Document downloaded from:

http://hdl.handle.net/10251/108675

This paper must be cited as:

Gómez-Tejedor, J.; Oset, E. (1996). Double pion photoproduction on the nucleon: study of the isospin channels. Nuclear Physics A. 600(4):413-435. doi:10.1016/0375-9474(95)004920

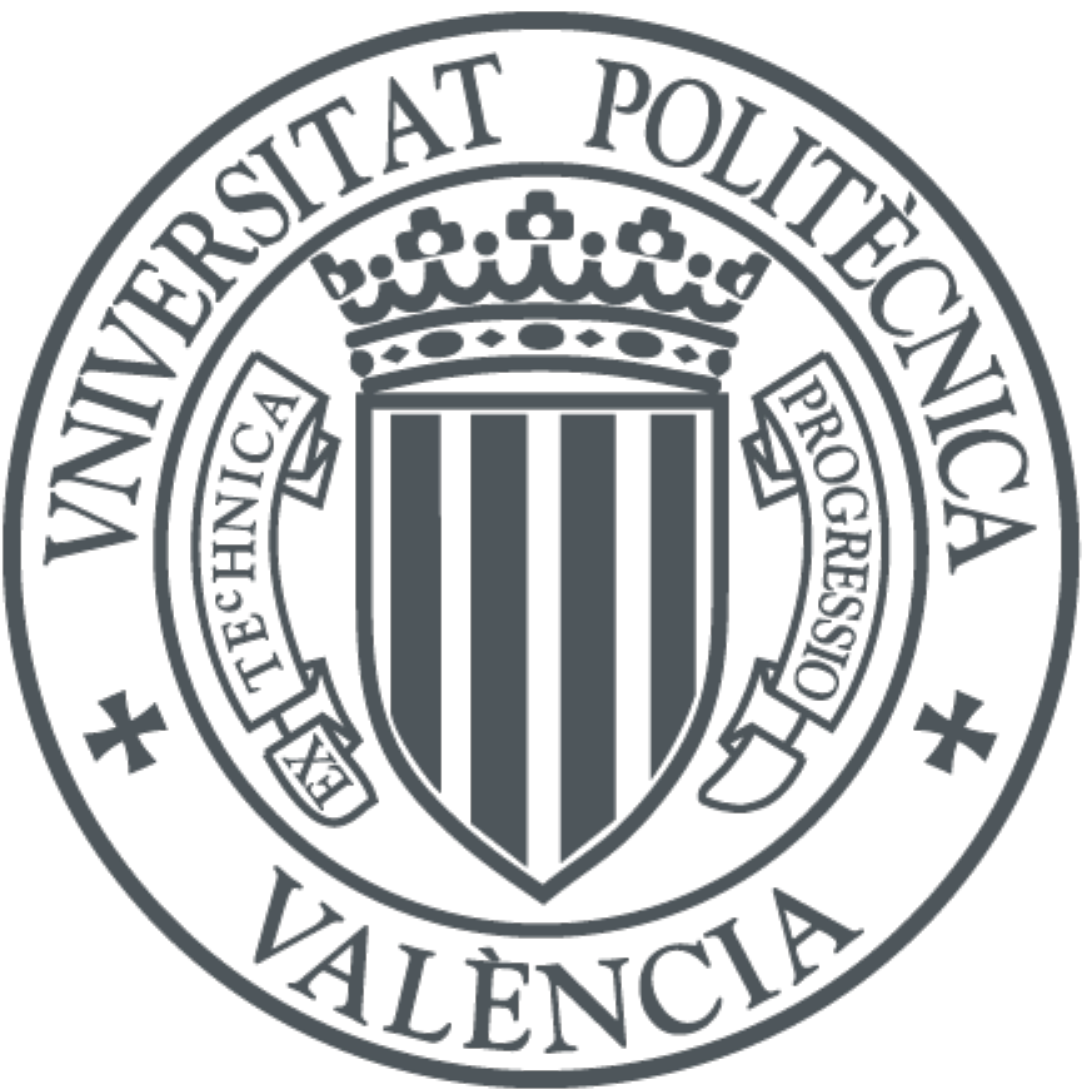

The final publication is available at

http://doi.org/10.1016/0375-9474(95)00492-0

Copyright Elsevier

Additional Information 


\title{
DOUBLE PION PHOTOPRODUCTION ON THE NUCLEON: STUDY OF THE ISOSPIN CHANNELS
}

\author{
J. A. Gómez Tejedor and E. Oset \\ Departamento de Física Teórica and IFIC, Centro Mixto Universidad de Valencia - \\ CSIC_— 46100 Burjassot (Valencia), Spain
}

\begin{abstract}
A model for the $\gamma p \rightarrow \pi^{+} \pi^{-} p$ reaction developed earlier is extended to account for all isospin channels. The model includes $N, \Delta(1232), N^{*}(1440)$ and $N^{*}(1520)$ as intermediate baryonic states and the $\rho$-meson as an intermediate $2 \pi$ resonance. Although many terms contribute to the cross section, some channels exhibit particular sensitivity to certain mechanisms of resonance excitation or decay and the reactions provide novel information on such mechanisms. In particular the $\gamma N \rightarrow N^{*}(1520) \rightarrow \Delta \pi$ process affects all the channels and is a key ingredient in the interpretation of the data. Comparison is made with all available data and the agreement is good in some channels. The remaining discrepancies in some other channels are discussed.
\end{abstract}




\section{Introduction.}

There are three possible double pion photoproduction reactions on the proton, and three on the neutron:
(a) $\gamma p \rightarrow \pi^{+} \pi^{-} p$
(b) $\gamma p \rightarrow \pi^{+} \pi^{0} n$
(d) $\gamma n \rightarrow \pi^{+} \pi^{-} n$
(c) $\gamma p \rightarrow \pi^{0} \pi^{0} p$
(e) $\gamma n \rightarrow \pi^{-} \pi^{0} p$
(f) $\gamma n \rightarrow \pi^{0} \pi^{0} n$

These reactions have been extensively studied experimentally in the past ([1, 2, 3, 4, 5, 6, 77). New improvements in experimental techniques and facilities have reopened the study of these reactions at Mainz, with two experiments on the proton [8, 9].

From the theoretical point of view, only the reaction $(a)$ has been studied with one early model [10] which considers only 5 Feynman diagrams, and a more complete one [11, 12] which includes $N, \Delta(1232), N^{*}(1440)$ and $N^{*}(1520)$ as intermediate baryonic states and the $\rho$-meson as intermediate $2 \pi$-resonance. This model reproduces fairly well the experimental cross sections below $E_{\gamma}=800 \mathrm{MeV}$, and the invariant mass distributions even at higher energies [11].

On the other hand, the double pion photoproduction reactions on nucleons has been recently studied at threshold from the point of view of chiral perturbation theory [13, 14, 15, 16]. In ref. [14] the authors show that due to finite chiral loops the cross section at threshold for final states with two neutral pions is considerably enhanced.

Thus, with these reactions becoming a target of new experimental and theoretical studies, plus interesting medium effects predicted for the $\left(\gamma, \pi^{+} \pi^{-}\right)$reaction in nuclei [17] (in analogy to those already found for the $(\pi, 2 \pi)$ reaction [18, 19]), a thorough theoretical study of the $\gamma N \rightarrow \pi \pi N$ reaction is timely and opportune.

In this work, we have improved the model of reference [11], and have extended it to the other isospin channels of Eq. (11). Moreover, although is not the purpose of this paper to perform a thorough analysis of the $\gamma N \rightarrow \pi \pi N$ reaction at threshold, we have calculated the total cross section for the $\gamma p \rightarrow \pi^{+} \pi^{-} p$ and $\gamma p \rightarrow \pi^{0} \pi^{0} p$ reactions at threshold energies, and compared our result with the result given in ref. [13, 14] based on Chiral Perturbation Theory.

The model is based on the coupling of photons and pions to nucleons and resonances using effective Lagrangians and thus leading to a set of Feynman diagrams at the tree level. As well as in ref. [11], we do not implement unitarity in the final states, but we made an estimate of possible uncertainties owing to unitary corrections for the $\gamma p \rightarrow \pi^{+} \pi^{-} p$ channel.

\section{The model for the $\gamma N \rightarrow \pi \pi N$ reaction.}

\section{1 the $\gamma p \rightarrow \pi^{+} \pi^{-} p$ channel.}

For the $\gamma p \rightarrow \pi^{+} \pi^{-} p$ isospin channel we follow strictly the model of ref. [11], with some slight modifications in the $N^{*}(1440)$ and $N^{*}(1520)$ couplings in order 
to reproduce the new branching ratios of the last Review of Particle Properties [20] (see Appendix A of the present paper for Lagrangians and coupling constants), and we have also improved the $N^{*}(1440)$ propagator, modifying the energy dependence of the width to account explicitly for the $N^{*}(1440)$ decay into one and two pions.

We classify our diagrams in one point, two point and three point diagrams, according to the number of vertices in the baryonic lines (see Fig. 1). Our basic components are pions, nucleons and nucleonic resonances: For the baryonic components we consider $N, \Delta\left(1232, J^{\pi}=3 / 2^{+}, I=3 / 2\right), N^{*}\left(1440, J^{\pi}=1 / 2^{+}, I=\right.$ $1 / 2)$ and $N^{*}\left(1520, J^{\pi}=3 / 2^{-}, I=1 / 2\right)$. The $N^{*}(1440)$ played an important role in the $\pi N \rightarrow \pi \pi N$ reaction [18, 19] in some isospin channels and for this reason, together with the fact that the $N^{*}(1440)$ excited in the $\gamma N$ collision is on-shell for $E_{\gamma} \simeq 600 \mathrm{MeV}$, we pay a special attention to this resonance. The $N^{*}(1520)$ has a particularly large coupling to the photons and proves to be an important ingredient, mostly due to its interference with the dominant term of the process, the $\gamma N \rightarrow \Delta \pi$ transition through the gauge Kroll-Ruderman term. Higher resonances have a weaker coupling to photons and do not interfere with the dominant term, hence their contribution is small, at least for photon energies below $800 \mathrm{MeV}$, Mainz energies, where our model is supposed to work. Because of the important coupling of the $\rho$-meson to the two pion system and the $\gamma \pi$ system we have also considered terms involving the $\rho$-meson. These terms are only relevant at high energies but show up clearly in the two pion invariant mass distributions at these energies [11].

With these considerations, the basic diagrams which we consider have the structure shown in Fig. 1. In diagram (a) and (b) the one point $N N \pi \pi$ coupling stands for the $s$-wave $\pi N$ interaction. We consider there only the isoscalar part of the amplitude. The isovector part is mediated by $\rho$ exchange [21] and hence it is explicitly taken into account in diagrams (f) and (h). Diagram (c) contains the gauge term $N N \pi \gamma$ or Kroll-Ruderman term. We use a pseudovector coupling for the $N N \pi$ vertex and this allows us to consider exclusively positive energy intermediate states in the hadronic propagators [22]. In the two point and three point diagrams we include nucleon and the resonances as intermediate states. However, while all possible diagrams with $\mathrm{N}$ and $\Delta(1232)$ intermediate states are considered, we omit some with $N^{*}(1440)$ intermediate states which are very small. For the $N^{*}(1520)$ intermediate states we keep only the term which interferes with the dominant term of the amplitude $(\Delta(1232)$ Kroll-Ruderman term $)$. In addition, all different time orderings of the diagrams are considered.

The diagram ( $\mathrm{g}$ ) involves a gauge term $\rho \pi \pi \gamma$ coming from minimal coupling in the $\rho \pi \pi$ vertex which contains a derivative coupling. The diagram (i) involves the $\gamma \rho \pi$ vertex which appears in the $\rho \rightarrow \gamma \pi$ decay. Finally diagram (j) contains the anomalous coupling $\gamma 3 \pi$ [23].

The Feynman diagrams considered and detailed calculations can be found in refs. [11, 12].

In spite of the small contribution of the $N^{*}(1440)$ terms in the reaction $\gamma p \rightarrow$ 
$\pi^{+} \pi^{-} p$ (less than $2 \%$ [11])円, in order to be consistent we have revised the value of the coupling constants involving the $N^{*}(1440)$ so that one may fit the new branching ratios of ref. [20] (see Appendix A of the present paper for coupling constants). This updating gives us very small differences compared to the values given in ref. [11].

The main change appears in the $N^{*} \Delta \pi$ coupling constant due to the fact that in the study of the decay of $N^{*}(1440)$ into $\Delta \pi$ of ref. [11] we considered the $\Delta(1232)$ as a stable particle. Now we modify this in order to take into account the finite width of the $\Delta(1232)$. The fact that the $\Delta(1232)$ width is not small compared to the mass difference between the $N^{*}(1440)$ and the $\Delta(1232)$ makes this correction advisable.

Making use of the Lagrangian (A.7), the decay width in the c.m. system of the $N^{*}(1440)$ into $\Delta \pi$, considering the $\Delta(1232)$ as a stable particle (zero width), is given by:

$$
\Gamma_{N^{*} \rightarrow \Delta \pi}=\frac{1}{(2 \pi)^{2}} \frac{g_{\Delta N^{*} \pi}^{2}}{\mu^{2}} \int d^{3} p \frac{m_{\Delta}}{E_{\Delta}(\vec{p})} \frac{1}{2 \omega(\vec{p})} \frac{4}{3} \vec{p}^{2} \delta\left(m_{N^{*}}-E_{\Delta}(\vec{p})-\omega(\vec{p})\right)
$$

where $\mu, m_{\Delta}$ and $m_{N^{*}}$ are the pion, $\Delta(1232)$ and $N^{*}(1440)$ masses respectively; $\vec{p}$ is the momentum of the outgoing pion; $E_{\Delta}(\vec{p})=\sqrt{m_{\Delta}^{2}+\vec{p}^{2}}$ and $\omega(\vec{p})=$ $\sqrt{\mu^{2}+\vec{p}^{2}}$ are the $\Delta(1232)$ and pion energies.

In order to account for the finite $\Delta(1232)$ width, we have to replace the delta function of energy conservation in Eq. (2) by

$$
\frac{-1}{\pi} \operatorname{Im} \frac{1}{m_{N^{*}}-E_{\Delta}(\vec{p})-\omega(\vec{p})+\frac{i}{2} \Gamma_{\Delta}\left(\sqrt{s_{\Delta}}\right)}
$$

where $\Gamma_{\Delta}\left(\sqrt{s_{\Delta}}\right)$ is the delta decay width, and $\sqrt{s_{\Delta}}$ is the $\Delta$ invariant mass, $\sqrt{s_{\Delta}}=$ $\sqrt{p_{\Delta}^{0}{ }^{2}-\vec{p}_{\Delta}^{2}}\left(p_{\Delta}=\left(p_{\Delta}^{0}, \vec{p}_{\Delta}\right)\right.$ is the $\Delta$ four momentum). With this modification, and using for $\Gamma_{\Delta}$ the expression (B.32) of ref. [11], we get $g_{\Delta N^{*} \pi}=2.07$ after fitting the $N^{*}(1440)$ decay width into $\Delta \pi$ to the average experimental value $(87.5$ $M e V)[20]$.

On the other hand, in the $N^{*}(1440)$ propagator

$$
\frac{1}{\sqrt{s_{N^{*}}}-m_{N^{*}}+\frac{i}{2} \Gamma_{N^{*}}\left(\sqrt{s_{N^{*}}}\right)} \frac{m_{N^{*}}}{E_{N^{*}}\left(\vec{p}_{N^{*}}\right)}
$$

where $\sqrt{s_{N^{*}}}=\sqrt{{p_{N^{*}}^{0}}^{2}-\vec{p}_{N^{*}}{ }^{2}}$ is the $N^{*}(1440)$ invariant mass $\left(p_{N^{*}}=\left(p_{N^{*}}^{0}, \vec{p}_{N^{*}}\right)\right.$ is the $N^{*}(1440)$ four momentum), and $\Gamma_{N^{*}}\left(\sqrt{s_{N^{*}}}\right)$ is the $N^{*}(1440)$ decay width.

We have included in the $N^{*}(1440)$ decay width, both the decay into $N \pi$ and $N \pi \pi$, thus

$$
\Gamma_{N^{*}}\left(\sqrt{s_{N^{*}}}\right)=\Gamma_{N^{*} \rightarrow N \pi}\left(\sqrt{s_{N^{*}}}\right)+\Gamma_{N^{*} \rightarrow N \pi \pi}\left(\sqrt{s_{N^{*}}}\right)
$$

\footnotetext{
${ }^{1}$ However, for the case of photo-production of two neutral pions, the contribution of these terms is more relevant (between $10 \%$ and $60 \%$, depending on the energy), thus it is important to treat them carefully.
} 
where $\Gamma_{N^{*} \rightarrow N \pi}\left(\sqrt{s_{N^{*}}}\right)$ is the $N^{*}(1440)$ decay width into $N \pi$ which is given by

$$
\Gamma_{N^{*} \rightarrow N \pi}\left(\sqrt{s_{N^{*}}}\right)=\frac{3}{2 \pi}\left(\frac{\tilde{f}}{\mu}\right)^{2} \frac{m}{m_{N^{*}}}|\vec{q}|^{3} \theta\left(\sqrt{s_{N^{*}}}-m-\mu\right)
$$

where $\tilde{f}$ is the $N^{*}(1440) N \pi$ coupling constant (see Appendix A), and $|\vec{q}|$ is the pion momentum in the $N^{*}(1440)$ centre of mass system. We take a fraction of $65 \%$ for the decay into $N \pi$ [20]. $\Gamma_{N^{*} \rightarrow N \pi \pi}\left(\sqrt{s_{N^{*}}}\right)$ is the $N^{*}(1440)$ decay width into $N \pi \pi$, which we approximate by a constant, multiplied by three body phase space. This constant is fitted in order to reproduce the experimental $N^{*}(1440)$ decay width into $N \pi \pi$. Although part of the $N^{*} \rightarrow N \pi \pi$ decay goes through the $\Delta \pi$ channel (and this is explicitly considered in the Feynman diagrams) we follow the above prescription solely for the purpose of providing the energy dependence of the $N^{*}(1440)$ width.

Apart from these small changes in the $N^{*}(1440)$ terms, the main improvement of the model of ref. [11] is coming from the modification of the $N^{*}(1520) \Delta \pi$ coupling. In ref. [11] we took the simplest Lagrangian compatible with the conservation laws:

$$
\mathcal{L}_{N^{\prime *} \Delta \pi}=i \tilde{f}_{N^{\prime *} \Delta \pi} \bar{\Psi}_{N^{\prime *}} \phi^{\lambda} T^{\lambda} \Psi_{\Delta}+\text { h.c. }
$$

where $\Psi_{N^{\prime *}}, \phi^{\lambda}$ and $\Psi_{\Delta}$ stand for the $N^{*}(1520)$, pion and $\Delta(1232)$ fields $\left(N^{*}\right.$ stands for the $N^{*}(1520)$ in the formulae from now on); $T^{\lambda}$ is the transition isospin operator from $1 / 2$ to $3 / 2$ with the normalization in terms of a Clebsch-Gordan coefficient

$$
\left\langle\frac{3}{2}, M\left|T_{\nu}^{\dagger}\right| \frac{1}{2}, m\right\rangle=C\left(\frac{1}{2}, 1, \frac{3}{2} ; m, \nu, M\right)
$$

and $\tilde{f}_{N^{\prime *} \Delta \pi}$ is the coupling constant, that we fixed from the data of $N^{*}(1520)$ decay into $\Delta \pi$ of ref. [24]. The sign however was chosen such as to have constructive interference below the $N^{*}(1520)$ pole. With the chosen sign the agreement with the data was relatively good, while with the opposite sign the discrepancies were about a factor of two and the qualitative features of the experiment were not reproduced [11].

Here, it is important to note that the $N^{*}(1520)$ can decay into $\Delta \pi$ both in $s$ wave and $d$-wave [20]. However, the Lagrangian of Eq. (7) gives only the decay into $s$-wave, not $d$-wave't Then, we have modified the Lagrangian of Eq. (7) in order to get a term which gives a contribution to the $d$-wave decay:

$$
\mathcal{L}_{N^{\prime *} \Delta \pi}=i \bar{\Psi}_{N^{\prime *}}\left(\tilde{f}_{N^{\prime *} \Delta \pi}-\frac{\tilde{g}_{N^{\prime *} \Delta \pi}}{\mu^{2}} S_{i}^{\dagger} \partial_{i} S_{j} \partial_{j}\right) \phi^{\lambda} T^{\lambda} \Psi_{\Delta}+\text { h.c. }
$$

\footnotetext{
${ }^{2}$ In the previous version of the Review of Particle Properties [24], there were not branching ratios to $s$-wave and $d$-wave, and only the total decay into $\Delta \pi$ was given. Thus, in ref. [11] we assumed that all the decay was through the $s$-wave, and we neglected the $d$-wave. As the fraction of decay into the $d$-wave is even bigger than into the $s$-wave [20], corrections to the coupling of Eq. (7) must be now implemented. The influence of these changes in the results for the $\gamma N \rightarrow \pi \pi N$ reaction, although not large, certainly help to improve agreement with the data, as we shall see.
} 
which gives us the vertex contribution:

$$
-i \delta H_{N^{\prime *} \Delta \pi}=-\left(\tilde{f}_{N^{\prime *} \Delta \pi}+\frac{\tilde{g}_{N^{\prime *} \Delta \pi}}{\mu^{2}} \vec{S}^{\dagger} \cdot \vec{q} \vec{S} \cdot \vec{q}\right) T^{\lambda}
$$

where $\vec{S}$ is the $1 / 2$ to $3 / 2$ spin transition operators with the same normalization as Eq. (8), $\mu$ is the pion mass and $\vec{q}$ is the pion momentum in the $N^{*}(1520)$ rest frame.

In order to fit the coupling constants to the experimental $N^{*}(1520)$ decay amplitudes to $\Delta \pi$ in $s$ - and $d$-wave [20, 25], we make a partial wave expansion [26] of the transition amplitude from a state of spin $3 / 2$ and third component $\mathrm{M}$, to a state of spin $3 / 2$ and third component $\mathbf{M}^{\prime}$ :

$$
\begin{array}{r}
-i\left\langle\frac{3}{2} M^{\prime}\left|\delta H_{N^{\prime *} \Delta \pi}\right| \frac{3}{2}, M\right\rangle=A_{s} Y_{0}^{M-M^{\prime}}(\theta, \phi)+ \\
A_{d} C\left(2, \frac{3}{2}, \frac{3}{2} ; M-M^{\prime}, M^{\prime}, M\right) Y_{2}^{M-M^{\prime}}(\theta, \phi)
\end{array}
$$

where $Y_{l}^{m}(\theta, \phi)$ are the spherical harmonics, and $A_{s}$ and $A_{d}$ are the $s$ - and $d$-wave partial amplitudes for the $N^{*}(1520)$ decay into $\Delta \pi$. In order to compare with the experimental signs of the amplitudes [20, 25] in Eq. (11) we have followed the standard "baryon-first" phase convention [27]. In this convention, spin couplings in all angular momentum Clebsch-Gordan coefficients are ordered such that the orbital angular momentum $L$ comes before the intrinsic spin $S$. The angles in the spherical harmonics are those of the pion referred to the baryon. Then, making use of the coupling of Eq. (10), the $A_{s}$ and $A_{d}$ amplitudes are given by:

$$
\begin{aligned}
& A_{s}=-\sqrt{4 \pi}\left(\tilde{f}_{N^{\prime *} \Delta \pi}+\frac{1}{3} \tilde{g}_{N^{\prime *} \Delta \pi} \frac{\vec{q}^{2}}{\mu^{2}}\right) \\
& A_{d}=\frac{\sqrt{4 \pi}}{3} \tilde{g}_{N^{\prime *} \Delta \pi} \frac{\vec{q}^{2}}{\mu}
\end{aligned}
$$

By using Eq. (11) we obtain the $N^{*}(1520)$ width into $\Delta \pi$ :

$$
\Gamma_{N^{\prime *} \rightarrow \Delta \pi}=\frac{1}{4 \pi} \frac{m_{\Delta}}{m_{N^{\prime *}}} q\left(\left|A_{s}\right|^{2}+\left|A_{d}\right|^{2}\right)
$$

where $m_{\Delta}$ and $m_{N^{*}}$ are the $\Delta$ and $N^{*}(1520)$ masses respectively, and $q$ is the momentum of the outgoing pion in the $N^{*}(1520)$ rest frame. Now, we fit the $N^{*}(1520)$ partial decay width in $s$ - and $d$-wave to the experiment [20] (we take the average values for these partial decays, $8.5 \%$ for the $s$-wave, and $12 \%$ for the $d$-wave). In addition, we impose the ratio $A_{s} / A_{d}$ to be positive, as deduced from the experimental analysis of the $\pi N \rightarrow \pi \pi N$ reaction [25]. Thus, with this last restriction, we get two different solutions for the coupling constants which differ only in a global sign:

(a) $\quad \tilde{f}_{\tilde{N}^{\prime *} \Delta \pi}=0.911 \quad \tilde{g}_{N^{\prime *} \Delta \pi}=-0.552$

(b) $\quad \tilde{f}_{N^{\prime *} \Delta \pi}=-0.911 \quad \tilde{g}_{N^{\prime *} \Delta \pi}=0.552$

Now, the $\gamma p \rightarrow \pi^{+} \pi^{-} p$ reaction allows us to distinguish between both solutions. In Fig. 2 we have plotted the total cross section for both solutions. As we can 
see, only solution $(a)$ fits the experiment, while the other one under-estimates the experimental cross section by a large amount.

This strong dependence of the total cross section on the $N^{*}(1520)$ coupling was already remarked in ref. [11], and it is due to the important interference between the $\Delta$ Kroll-Ruderman term (diagram $(i)$ of Fig. 3) and the $\gamma p \rightarrow N^{*}(1520) \rightarrow$ $\Delta \pi$ term (diagram (p) of Fig. 3) in $s$-wave [11]. The amplitudes for these two diagrams have exactly the same structure, except by the $N^{*}(1520)$ propagator and coupling constants (see Eq. (6) of ref. [1]], where $\tilde{f}_{N^{\prime *} \Delta \pi}$ would be substituted by $\left.\tilde{f}_{N^{\prime *} \Delta \pi}+\frac{1}{3} \tilde{g}_{N^{\prime *} \Delta \pi} \vec{q}^{2} / \mu^{2}\right)$. Then, the $N^{*}(1520)$ interference term is proportional to the real part of the $N^{*}(1520)$ propagator [11]. There is also a contribution to the total cross section coming from the imaginary part of the $N^{*}(1520)$ propagator, and also from the $A_{d}$ amplitude, which do not interfere. Thus, this interference is zero at $\sqrt{s}=m_{N^{\prime *}}$, where the real part of the $N^{*}(1520)$ propagator vanishes. Then, what matters in the interference is the value of $A_{s}$ for different values of $q$ than the one from the $N^{*}(1520)$ on-shell. Then, the $\gamma p \rightarrow \pi^{+} \pi^{-} p$ reaction provides us with novel information about the $q$ dependence of the $A_{s}$ amplitude, respect to the one obtained from the analysis of the $\pi N \rightarrow \pi \pi N$ reaction [25].

This $q$ dependence in the $s$-wave amplitude is given by the chosen Lagrangian (on the other hand for the $A_{d}$ amplitude there is no choice in the momentum dependence exhibited in Eq. (12)). It is, thus, possible to postulate other Lagrangians which would produce a different $q$ dependence of the $A_{s}$ amplitude. For instance, in a preliminary work reported in [28, 29] a different Lagrangian was investigated. There we proposed a similar Lagrangian to Eq. (9), but with the 3/2 spin operators instead of the $S^{i}$ transition operators. With such a Lagrangian and an appropriate choice of parameters we were able to reproduce the experimental data, though the ratio $A_{s} / A_{d}$ was negative.

In order to investigate the most general $q$ dependence of the amplitude, we replace $\tilde{f}_{N^{\prime *} \Delta \pi}$ by

$$
\tilde{f}_{N^{\prime *} \Delta \pi}\left(1+\epsilon \frac{\vec{q}^{2}-\vec{q}_{o n-s h e l l}^{2}}{\mu^{2}}\right)
$$

where $\vec{q}$ is the momentum of the decay pion, and $\vec{q}_{\text {on-shell }}^{2}$ is de momentum of the pion for an on-shell $N^{*}(1520)$ decaying into $\Delta \pi\left(\left|\vec{q}_{\text {on-shell }}\right|=228 \mathrm{MeV}\right)$. Then, we change $\epsilon$ and compare the results to the data. We find that, to a good approximation, $\epsilon=0$ gives the best agreement with the data, hence supporting the Lagrangian of Eq. (9).

It is interesting to remark here that this $q$ dependence of the amplitudes coincides exactly with the predictions of the non-relativistic constituent quark models, although the absolute values of the amplitudes are only qualitatively given by these models [30].

\subsection{The others isospin channels.}

For the others isospin channels we use the same model as for the $\gamma p \rightarrow \pi^{+} \pi^{-} p$ case, changing the isospin factors and introducing some terms which are only relevant in 
the case of neutral pions (although their influence in the cross sections is small). However, we have neglected some Feynman diagrams which were found to be very small in [11]. The Feynman diagrams that we take into account are shown in Fig. 3 , where all possible charge combinations allowed by charge conservation have to be implemented for each isospin channel. In the case of neutral pions and neutral deltas some of the diagrams in Fig. 3 are automatically zero. For instance, diagrams (c) and (d) of Fig. 3 with the photon attached to a neutral pion are zero.

Nevertheless, some remarks are necessary for the isospin channels with neutral pions: When in the diagrams of Fig. 4 (a) and (b) we evaluate the contribution from the intermediate states of negative energy we get an extra contribution with the same structure as the Kroll-Ruderman term [22]. In the case of charged pions, we have neglected this contribution due to the fact that it is very small compared with the Kroll-Ruderman terms. However, for the neutral pions, as there is no KrollRuderman term, this contribution has to be considered and, for a nucleon in the final state is given by [22]:

$$
-i T_{\alpha}=e \frac{f}{\mu} C(\alpha) \vec{\sigma} \cdot \vec{\varepsilon}
$$

where

$$
C(\alpha)= \begin{cases}-q^{0}\left(\frac{1}{p^{0}+k^{0}+E(\vec{p}+\vec{k})}+\frac{1}{p^{0}-k^{0}+E\left(\vec{p}^{\prime}-\vec{k}\right)}\right) & \text { for proton } \\ 0 & \text { for neutron }\end{cases}
$$

where $\left(p^{0}, \vec{p}\right),\left({p^{\prime}}^{0}, \vec{p}^{\prime}\right),\left(q^{0}, \vec{q}\right)$ and $\left(k^{0}, \vec{k}\right)$ are the four-momentum of the incoming nucleon, the outgoing nucleon, the pion and the photon respectively.

We can write Eq. (16) as:

$$
-i T=\frac{1}{2}\left(1+\left\langle N\left|\tau_{3}\right| N\right\rangle\right) e \frac{f}{\mu} C \vec{\sigma} \cdot \vec{\varepsilon}
$$

where

$$
C=-q^{0}\left(\frac{1}{p^{0}+k^{0}+E(\vec{p}+\vec{k})}+\frac{1}{p^{\prime 0}-k^{0}+E\left(\vec{p}^{\prime}-\vec{k}\right)}\right)
$$

and $\tau_{3}$ is the third component of the isospin Pauli matrices:

$$
\left\langle p\left|\tau_{3}\right| p\right\rangle=1 \quad ; \quad\left\langle n\left|\tau_{3}\right| n\right\rangle=-1
$$

The advantage of expression (18) is that it allows us to generalize it to the case where we have a $N^{*}(1440)$ or a $\Delta(1232)$ in the final state by the following method: for the $N^{*}(1440)$ we replace the coupling constant $N N \pi(f)$ by the coupling constant $N^{*} N \pi(\tilde{f})$ :

$$
-i T=\frac{1}{2}\left(1+\left\langle N\left|\tau_{3}\right| N\right\rangle\right) e \frac{\tilde{f}}{\mu} C \vec{\sigma} \cdot \vec{\varepsilon}
$$


For the $\Delta(1232)$ case we replace the coupling constant $N N \pi(f)$ by the $\Delta N \pi$ coupling constant $\left(f^{*}\right)$ and the $\vec{\sigma}$ and $\tau_{3}$ operators by the transition spin an isospin operators from $1 / 2$ to $3 / 2$ objects (with the normalization given by Eq. (8)). However, the isoscalar part does not contribute in this case. Then, for the $\Delta N \pi^{0}$ coupling we have:

$$
-i T=\frac{1}{2} e \frac{f^{*}}{\mu} C \vec{S}^{\dagger} \cdot \vec{\varepsilon} T_{3}^{\dagger}
$$

The vertices $\gamma N N \pi^{0}, \gamma N^{*} N \pi^{0}$ and $\gamma \Delta N \pi^{0}$ of Eqs. (18), (21) and (22) are diagrammatically represented with the same Feynman graphs as the corresponding Kroll-Ruderman terms with charged pions in Fig. 3.

The effective Lagrangians needed for the evaluation of the Feynman diagrams and the corresponding coupling constants are given in appendix A. The corresponding Feynman rules are given in Appendix B of ref. [11].

\section{Results and discussions.}

In Figs. 5 to 10 we show the total cross sections for all the isospin channels, as well as the contribution to the total cross section of diagrams with nucleon, $\Delta(1232)$, $N^{*}(1440), N^{*}(1520)$ and $\rho(770)$ as intermediate states (in ref. [11] we also showed differential cross sections and invariant-mass distributions for the $\gamma p \rightarrow \pi^{+} \pi^{-} p$ channel). We show results up to $E_{\gamma}=800 \mathrm{MeV}$, where the new experiments at Mainz concentrate, and we compare our results with the available experimental data [1], 2, 3, 4, 8]. In the errorbars of the DAPHNE data [8] we have plotted only the statistical errors. The systematic errors are of the same order of magnitude.

The isospin channels with one or two charged pions in the final state are shown in Figs. 5, 6, 8 and 9. We observe that the $\Delta(1232)$ terms (short-dashed lines) are dominant in these isospin channels. Essentially the $\Delta(1232)$ Kroll-Ruderman and $\Delta(1232)$ pion-pole terms (diagrams (i) and (k) of Fig. 3) are the more important terms. The non resonant terms (short-dash-dotted lines) are much smaller and they provide a small background which grows up moderately as a function of the energy. The $N^{*}(1520)$ contribution (long-dashed lines) by itself is also small compared to the $\Delta(1232)$ one, but it is essential to reproduce the total cross section due to its interference with the $\Delta(1232)$ Kroll-Ruderman term as we already remarked in ref. [11]. This interference occurs only between the $\Delta(1232)$ Kroll-Ruderman term and the $s$-wave part of the $N^{*}(1520) \Delta \pi$ contribution. Since now part of the $N^{*} \rightarrow \Delta \pi$ decay is due to $d$-wave, the interference effects are smaller than in ref. [11] where all the $N^{*}(1520) \rightarrow \Delta \pi$ decay was associated to $s$-wave. This has as a consequence a better agreement of theory with experiment than in ref. [11] (see Fig. 5 here in comparison with Fig. 5 of ref. [11]). The $\rho$-terms are negligible at these energies, but they show up clearly at higher energies (see ref. [11]). Contributions from other terms are still smaller.

For the isospin channels with two neutral pions in the final state things are rather different (see Figs. 7 and 10). In these cases a lot of terms vanish due to 
the fact that the photons cannot couple to neutral pions. In particular, there are no Kroll-Ruderman and pion-pole terms. Thus, the total cross section for these isospin channels is much smaller than the cross section for the other isospin channels. Furthermore, terms that in the other isospin channels are very small become important in these isospin channels.

In Figs. 7 and 10 we can see that, except for the contribution from $\mathrm{N}$-intermediate states (short-dash-dotted lines), which is very small, the other contributions are all of them relevant. Below $500 \mathrm{MeV}$ we can see that the $N^{*}(1440)$ (long-dash-dotted lines) dominates the reactions. At $500 \mathrm{MeV}$ the $\Delta(1232)$ (short-dashed lines) and the $N^{*}(1520)$ (long-dashed lines) start to grow up, and around $600 \mathrm{MeV}$ all these diagrams have similar strength. At $600 \mathrm{MeV}$ the $N^{*}(1440)$ contribution starts to fall down, and the $N^{*}(1520)$ and $\Delta(1232)$ dominate the reaction. The $N^{*}(1520)$ contribution peaks at $720 \mathrm{MeV}$ (it is responsible of the peak of the total cross sections) and from this energy on it falls down, while the $\Delta(1232)$ contribution continues growing up moderately.

It is worth noting that, in these latter cases, the $N^{*}(1520)$ contribution is important by itself, and not by its interference with other terms as it happens in the isospin channels with one or two charged pions, and again it is essential to reproduce the peak of the cross section around $700 \mathrm{MeV}$. To further stress the role of the $N^{*}(1520)$ resonance, we plot in Fig. 11 the results for the $\gamma p \rightarrow \pi^{0} \pi^{0} p$ cross section using the two possible combinations of Eq. (14) for the $s$ - and $d$-wave couplings. We see that the curve $(a)$ is clearly favoured by the data, precisely the same case which was favoured in the $\gamma p \rightarrow \pi^{+} \pi^{-} p$ reaction. This provides further support for our choice of amplitudes in the $N^{*}(1520) \rightarrow \Delta \pi$ decay.

At this point, we should mention that in spite of the large $N^{*}(1440)$ decay branching ratio into $N \pi \pi$ (around 35\%), and the fact that in the present reaction the $N^{*}(1440)$ is on-shell at $E_{\gamma} \simeq 600 \mathrm{MeV}$, the $N^{*}(1440)$ contribution to the total cross section is very small for some of the isospin channels. This is due to the relatively small $N^{*}(1440)$ coupling to photons [20], compared to the $\Delta(1232)$ or the $N^{*}(1520)$ resonances (the branching ratio to $N \gamma$ is around one order of magnitude smaller for the $N^{*}(1440)$ than for the $\Delta(1232)$ or the $\left.N^{*}(1520)\right)$. However, it is important in the two-neutral pion channels in the low energy region (below $E_{\gamma} \simeq 650 \mathrm{MeV}$ ) (see long-dash-dotted line of Figs. 7 and 10). Note again, that for $E_{\gamma}<600 \mathrm{MeV}$ the $N^{*}(1440)$ is the dominant contribution, and although there are some discrepancies with de DAPHNE data [8] in this region, our model agrees well with the preliminary data of the TAPS collaboration [9] in the same region.

For the total cross sections, in Figs. 5 to 10 (solid lines) we can see a remarkable agreement with the experiment in some isospin channels, and some discrepancies in others.

For the $\gamma p \rightarrow \pi^{+} \pi^{-} p$ channel (Fig. 5) we reproduce quite well the total cross section up to $E_{\gamma}=800 \mathrm{MeV}$ (we also reproduce invariant mass distributions even at higher energies [11]]).

For the $\gamma p \rightarrow \pi^{+} \pi^{0} n$ channel (Fig. 6) we have found an important discrepancy between our calculations and the experimental data. It is very easy to understand qualitatively our results for the $\gamma p \rightarrow \pi^{+} \pi^{0} n$ cross section from isospin coeffi- 
cients. As we have already said, the photoproduction of one or two charged pions is dominated by the $\Delta(1232)$ Kroll-Ruderman and $\Delta(1232)$ pion-pole terms. In the $\gamma p \rightarrow \pi^{+} \pi^{-} p$ channel, these terms with a $\Delta^{++}$in the intermediate state have a factor 1 in the amplitude from isospin $\left(T^{\lambda}\right)$, while in the $\gamma p \rightarrow \pi^{+} \pi^{0} p$ case with a $\Delta^{0}$ in the intermediate state, this factor is $\sqrt{2} / 3$. Then we have that the cross section for the $\gamma p \rightarrow \pi^{+} \pi^{0} n$ channel is around 4.5 times smaller than the $\gamma p \rightarrow \pi^{+} \pi^{-} p$ one (this relation is not exact because there are other terms with different isospin coefficients that also contribute). As we can see comparing Figs. 5 and 6 this relation is approximately satisfied.

In order to understand these discrepancies, we have tried to look for additional contributions for this channel. For instance, we have calculated the contribution to the total cross section of new diagrams with $\rho$-intermediate state decaying into $\pi \pi$ where the photon is coupled to the $N N \rho$ vertex in one case and to the intermediate $\rho$ meson line in another case. Note that these additional diagrams only contribute for the $\gamma p \rightarrow \pi^{+} \pi^{0} n$ and $\gamma n \rightarrow \pi^{-} \pi^{0} p$ channels, because for the others channels, the intermediate $\rho$-meson is neutral, and does not couple to photons. However, we have found that these contributions are very small, and they do not modify our results.

On the other hand, we have also investigated the contribution to the total cross section of the off-shell part of the $\Delta$ interactions with photons and pions (see ref. [31]). For this purpose, we have evaluated diagram $(i)$ of Fig. 3, including the off-shell part of the $\Delta$-interaction. Then, from the off-shell parameters of the $\Delta$ interaction, we get an additional term which is very similar to the one obtained from diagram $(i)$ of Fig. 3 without the off-shell parameters, but it has a multiplicative factor $\left(\sqrt{s_{\Delta}}-m_{\Delta}\right) / 2 m_{\Delta}\left(\sqrt{s_{\Delta}}\right.$ is the $\Delta$ invariant mass, and $m_{\Delta}$ is the $\Delta$ mass). This factor is zero for a $\Delta$ on-shell, and in our range of energies, it changes between -0.05 and +0.07 , and it strongly peaks around zero (see Fig. 6 of ref. [[1]]). This is so, because the pion of the $\gamma \Delta N \pi$ vertex in Fig. 3 (i) picks up the necessary momentum to make the $\Delta$ closest to on-shell. When we perform the actual calculations the modifications from the consideration of these off-shell corrections are of the order of $1 \%$ for a broad range of the off-shell $Z$ parameter (we have checked it for several values of $Z$ between $-1 / 2$ and $1 / 2$ ). Then, we conclude that the offshell contribution of the $\Delta(1232)$ resonance to the total cross section is very small, and we can neglect it.

For the $\gamma p \rightarrow \pi^{0} \pi^{0} p$ channel (Fig. 7) we can see a good agreement with the experimental data above $650 \mathrm{MeV}$, but our results are below the experimental ones for $E_{\gamma}<650 \mathrm{MeV}$. We should also mention that there are already preliminary results for this channel from the TAPS collaboration [9], which for $E_{\gamma}<600 \mathrm{MeV}$ are below the DAPHNE data, and our model agrees very well with these preliminary results in this energy region. Nevertheless, we should wait for their final results.

For the $\gamma n \rightarrow \pi^{+} \pi^{-} n$ we can see in Fig. 8 that we approximately reproduce the experimental data below $650 \mathrm{MeV}$, but above this energy our model overestimates the experimental data.

For the $\gamma n \rightarrow \pi^{-} \pi^{0} p$ isospin channel (Fig. 9), the theory is a bit below the experimental results [3, 4 ], but the trend of the energy dependence is well reproduced.

However, in connection with the data of refs. [3, 4] for the $\gamma n \rightarrow \pi^{+} \pi^{-} n$ 
and $\gamma n \rightarrow \pi^{-} \pi^{0} p$ reaction, we should point out that there is some disagreement between the results of the same experiments for the $\gamma p \rightarrow \pi^{+} \pi^{-} p$ channel and the experimental results of ref. [1], 8] (see Fig. 5). One should then be cautious and wait for new measurements in these channels before extracting any conclusion from the present discrepancies of our model with these data.

In Fig. 10 we show the results of our model for the $\gamma n \rightarrow \pi^{0} \pi^{0} n$ channel for which there are not yet any experimental data.

Due to the interest raised on the two pion photoproduction at threshold [9, 13, 14, 15, 16], we have also calculated the total cross section for the $\gamma p \rightarrow \pi^{+} \pi^{-} p$ and $\gamma p \rightarrow \pi^{0} \pi^{0} p$ channels at energies near threshold. In Figs. 12 and 13 we show our results for the total cross section for these channels, compared to the results of ref. [13, 14] (in ref. [13] only the amplitude at threshold is given, not the total cross section. Then, we have taken that amplitude as a constant, and we have integrated it over the three body phase space).

In Fig. 12 we see the results for the $\gamma p \rightarrow \pi^{+} \pi^{-} p$ channel. There appear to be some discrepancies between the results of refs. [13] and [14] which are mostly due to the fact that we use the constant amplitude at threshold of ref. [13]. Indeed, if the same is done for the work of ref. [14] the results are very similar (see Fig. 9 of ref. [14]). This also means that energy dependent terms are important even very close to threshold.

Our results are above the results of refs. [13, 14]. We should note that in addition to the Chiral terms which contribute at threshold, and which we have in terms of our effective Lagrangians, there is one important contribution at threshold which is missed in the two other approaches. This corresponds to the diagram of Fig 3. (q) and the crossed one which is not drown there but is actually calculated in this channel. They correspond to $N^{*}(1440)$ excitation with decay into $\pi \pi$ in $s$-wave and $N$. These terms were also shown to be important at threshold in the $\pi N \rightarrow \pi \pi N$ reaction [18]. Its relevance in that reaction has been stressed in a recent paper [32] where the existence of an additional energy dependent term of unknown strength is pointed out.

The different threshold behaviour of the approaches of refs. [13, 14] and the present work, apart from some differences in the treatment of the $\Delta$ terms, is mostly due to the fact that we include the $N^{*}(1440)$ resonance. In addition there is a small contribution from the $N^{*}(1520)$ a few $M e V$ above threshold, and there are also other terms which vanish at threshold but contribute close to it.

We should also mention that in the region of interest to us, $450 \mathrm{MeV}<E_{\gamma}<$ $800 \mathrm{MeV}$, the cross section is dominated by resonance terms which are not provided by Chiral Perturbation Theory.

In Fig. 13 we show the threshold results of the $\gamma p \rightarrow \pi^{0} \pi^{0} p$ channel. We observe large discrepancies between the results of refs. [13] and [14] which are commented in refs. [15, 16]. Among other differences, the results of ref. [14] include loop corrections which are relatively important in this channel close to threshold [14]. Once again our results, which also miss the loop corrections, are bigger than in the two approaches of refs. [13, 14] for the same reasons discussed above. Our results at threshold also include the contribution of the crossed term of 
the $N^{*}(1440)$ diagram of Fig. 3(q), which is negligible at higher energies.

Furthermore, we should also stress that, like in the $\gamma p \rightarrow \pi^{+} \pi^{-} p$ channel, the terms which vanish at threshold dominate the reaction at large energies.

One limitation of our model is that we neglect unitarity. Unitarity for three particles in the final state is a nontrivial problem [33], and is not the purpose of this paper to deal with it. However, for the dominant channel $(\gamma N \rightarrow \pi \Delta[\pi N])$, due to the fact that the $\Delta$-width is implemented in the $\Delta$-propagators, our model approximately satisfies unitarity. In order to estimate the errors that unitarity could introduce, we follow the procedure of Olsson [34] to unitarize this channel by multiplying the $\Delta$-terms by a phase and requesting that the resulting amplitude, after adding the background, has the phase of the $\pi N$ amplitude. The angle $\phi$ needed for this phase, $e^{i \phi}$, is of the order of $10^{0}$. We have checked, for the $\gamma p \rightarrow \pi^{+} \pi^{-} p$ channel, that implementing this phase in the amplitude changes the results at the level of $3 \%$ at energies below $E_{\gamma}=800 \mathrm{MeV}$. Even increasing the angle $\phi$ to $20^{0}$ the changes are of the same order of magnitude. This result is due to the fact that, except for the already commented interference between the $N^{*}(1520)$ and the $\Delta$ Kroll-Ruderman term, the interference between the different terms of the amplitude is small.

This is only a rough approach to such a difficult problem, however it gives hints that the unitary corrections might be small in that energy range. This conclusion is also supported by another approximate scheme to unitarize the Born amplitudes used in [10]. An absorptive correction factor is used there which does not modify the cross section below $E_{\gamma}=800 \mathrm{MeV}$, although it produces a substantial reduction at much higher energies. Nevertheless a rigourous treatment of this problem would be welcome.

\section{Conclusions.}

We have constructed a model for the $\gamma N \rightarrow \pi \pi N$ reaction extending the model of ref. [11] for the $\gamma p \rightarrow \pi^{+} \pi^{-} p$ reaction to the rest of the isospin channels, including some improvements in the $\Delta(1232), N^{*}(1440)$ and $N^{*}(1520)$ couplings. Our model reproduces quite well the experimental results of refs. [11, 8, 9] below $E_{\gamma}=800 \mathrm{MeV}$ for the $\gamma p \rightarrow \pi^{+} \pi^{-} p$ and $\gamma p \rightarrow \pi^{0} \pi^{0} p$ isospin channels, but we have found some important discrepancies for the $\gamma p \rightarrow \pi^{+} \pi^{0} n$ channel. For the isospin channels on the neutron we have found also some discrepancies with the

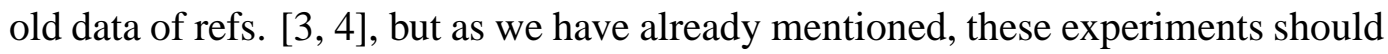
be improved.

As we already observed in ref. [11] the reaction is dominated by the $\Delta(1232)$ Kroll-Ruderman and $\Delta(1232)$ pion-pole terms, but we get an appreciable contribution from other terms. In particular we have shown the crucial importance of the $N^{*}(1520)$ terms. In the isospin channels with one or two charged pions in the final state, the $N^{*}(1520)$ contribution is small compared with the $\Delta(1232)$ terms, but it shows up clearly in the total cross section due to its interference with the $\Delta(1232)$ Kroll-Ruderman term. For the cases with two neutral pions in the final state the 
dominant terms in the other isospin channels vanish, and the contribution of the $N^{*}(1520)$ terms is bigger than the contribution of the $\Delta(1232)$. At energies below $E_{\gamma}=600 \mathrm{MeV}$ the $N^{*}(1440)$ plays an important role in the two neutral pion channels.

One of the interesting findings was the possibility to extract information on the coupling constants for $N^{*}(1520)$ decay into $\Delta \pi$. The interference of the $N^{*}(1520)$ excitation term, followed by $\Delta \pi$ decay, with the dominant $\Delta(1232)$ Kroll-Ruderman term in the $\gamma p \rightarrow \pi^{+} \pi^{-} p$ channel allowed us to determine the sign of the couplings for the $s$-wave and $d$-wave in the $N^{*}(1520) \rightarrow \Delta \pi$ decay, when the absolute values given by the branching ratios, and the constraint $A_{s} / A_{d}>0$ obtained form the $\pi N \rightarrow \pi \pi N$ reaction were used. We also observed that the same choice of amplitudes gave rise to a fair agreement with the $\gamma p \rightarrow \pi^{0} \pi^{0} p$ channel while the other possible choice gave rise to a cross section in sheer disagreement with the data.

While in the $\gamma n$ channels there are reasons to expect that the data will change when new experiments are done, the discrepancy found with the recent data [8] of the $\gamma p \rightarrow \pi^{+} \pi^{0} n$ channels is puzzling. For the moment we cannot envisage a solution to this problem. Alternative experiments would be in any case most welcome.

Acknowledgements: Discussions with M.J. Vicente-Vacas are warmly acknowledged. We would also like to thank N. d'Hose, G. Audit, G. Tamas and H. Ströher for multiple discussion around their preliminary data. This work has been partially supported by CICYT contract number AEN 93-1205. One of us J.A. Gómez Tejedor wishes to acknowledge financial support from the Institució Valenciana d'Estudis i Investigació. 


\section{LAGRANGIANS.}

\section{Appendix A}

$$
\mathcal{L}_{N N \pi}=\frac{-f}{\mu} \bar{\Psi} \gamma^{\mu} \gamma_{5} \partial_{\mu} \vec{\phi} \cdot \vec{\tau} \Psi
$$

$$
\mathcal{L}_{N N \pi \pi}=-4 \pi \frac{\lambda_{1}}{\mu} \bar{\Psi} \vec{\phi} \cdot \vec{\phi} \Psi
$$

$$
\mathcal{L}_{\Delta N \pi}=\frac{-f^{*}}{\mu} \Psi_{\Delta}^{\dagger} S_{i}\left(\partial_{i} \phi^{\lambda}\right) T^{\lambda} \Psi_{N}+\text { h.c. }
$$

$$
\mathcal{L}_{\Delta \Delta \pi}=\frac{-f_{\Delta}}{\mu} \Psi_{\Delta}^{\dagger} S_{\Delta i}\left(\partial_{i} \phi^{\lambda}\right) T_{\Delta}^{\lambda} \Psi_{\Delta}+\text { h.c. }
$$

$$
\mathcal{L}_{N^{*} N \pi}=\frac{-\tilde{f}}{\mu} \Psi_{N^{*}}^{\dagger} \sigma_{i}\left(\partial_{i} \vec{\phi}\right) \cdot \vec{\tau} \Psi_{N}+\text { h.c. }
$$

$$
\mathcal{L}_{N^{*} N \pi \pi}=-C \bar{\Psi}_{N^{*}} \vec{\phi} \cdot \vec{\phi} \Psi_{N}+\text { h.c. }
$$

$$
\mathcal{L}_{N^{*} \Delta \pi}=\frac{-g_{\Delta N^{*} \pi}}{\mu} \Psi_{\Delta}^{\dagger} S_{i}\left(\partial_{i} \phi^{\lambda}\right) T^{\lambda} \Psi_{N^{*}}+\text { h.c. }
$$

$$
\mathcal{L}_{N^{\prime *} \Delta \pi}=i \bar{\Psi}_{N^{\prime *}}\left(\tilde{f}_{N^{\prime *} \Delta \pi}-\frac{\tilde{g}_{N^{\prime *} \Delta \pi}}{\mu^{2}} S_{i}^{\dagger} \partial_{i} S_{j} \partial_{j}\right) \phi^{\lambda} T^{\lambda} \Psi_{\Delta}+\text { h.c. }
$$




$$
\mathcal{L}_{N N \gamma}=-e \bar{\Psi}_{N}\left(\gamma^{\mu} A_{\mu}-\frac{\chi_{N}}{2 m} \sigma^{\mu \nu} \partial_{\nu} A_{\mu}\right) \Psi_{N}
$$

$$
\mathcal{L}_{\pi \pi \gamma}=i e\left(\phi_{+} \partial^{\mu} \phi_{-}-\phi_{-} \partial^{\mu} \phi_{+}\right) A_{\mu}
$$

$$
\mathcal{L}_{N N \pi \gamma}=-i \mathrm{q} \frac{f}{\mu} \bar{\Psi} \gamma^{\mu} \gamma_{5} A_{\mu} \vec{\phi} \cdot \vec{\tau} \Psi
$$

$$
\mathcal{L}_{\Delta N \pi \gamma}=-i \mathrm{q} \frac{f^{*}}{\mu} \Psi_{\Delta}^{\dagger} S_{i} A_{i} \phi^{\lambda} T^{\lambda} \Psi_{N}+\text { h.c. }
$$

$$
\mathcal{L}_{N^{*} N \pi \gamma}=-i \mathrm{q} \frac{\tilde{f}}{\mu} \mathrm{q} \Psi_{N^{*}}^{\dagger} \sigma_{i} A_{i} \vec{\phi} \cdot \vec{\tau} \Psi_{N}+\text { h.c. }
$$

$$
\mathcal{L}_{\Delta N \gamma}=\frac{-f_{\Delta N \gamma}}{\mu} \Psi_{\Delta}^{\dagger} \epsilon_{i j k} S_{i}^{\dagger}\left(\partial_{j} A_{k}\right) T_{3} \Psi_{N}+\text { h.c. }
$$

$$
\mathcal{L}_{N^{*} N \gamma}=\frac{\tilde{f}_{\gamma}^{N}}{\mu} \bar{\Psi}_{N} \sigma^{\mu \nu} \partial_{\nu} A_{\mu} \Psi_{N^{*}}+\text { h.c. }
$$

$$
\mathcal{L}_{N^{*} N \gamma}=\bar{\Psi}_{N}\left\{\tilde{g}_{\gamma} \vec{S} \cdot \vec{A}-i \tilde{g}_{\sigma}(\vec{\sigma} \times \vec{S}) \cdot \vec{A}\right\} \Psi_{N^{*}}+\text { h.c. }
$$

$$
\mathcal{L}_{N N \rho}=-\bar{\Psi}\left\{G_{N N \rho}^{V} \gamma^{\mu} \vec{\phi}_{\mu}^{(\rho)}-\frac{G_{N N \rho}^{T}}{2 m} \sigma^{\mu \nu} \partial_{\nu} \vec{\phi}_{\mu}^{(\rho)}\right\} \cdot \vec{\tau} \Psi
$$




$$
\begin{aligned}
& \mathcal{L}_{\rho \pi \pi}=-f_{\rho} \vec{\phi}_{\mu}^{(\rho)} \cdot\left(\vec{\phi} \times \partial^{\mu} \vec{\phi}\right) \\
& \mathcal{L}_{\Delta N \rho}=-\sqrt{C_{\rho}} \frac{f^{*}}{\mu} \Psi_{\Delta}^{\dagger} \epsilon_{i j k} S_{i}\left(\partial_{j} \phi_{k}^{(\rho)}\right) T^{\lambda} \Psi+h . c . \\
& \mathcal{L}_{N^{\prime *} N \rho^{0}}=-\tilde{g}_{\rho} \bar{\Psi}_{N} S_{i} \phi_{i}^{(\rho)} \Psi_{N^{\prime *}}+h . c . \\
& \mathcal{L}_{\rho \pi \gamma}=\frac{g_{\rho \pi \gamma}}{\mu} \epsilon^{\alpha \beta \gamma \delta} \partial_{\alpha} A_{\beta} \vec{\phi} \partial_{\gamma} \vec{\phi}_{\delta}^{(\rho)} \\
& \mathcal{L}_{\rho^{0} \pi^{+} \pi^{-} \gamma}=e f_{\rho} \phi_{\mu}^{(\rho)}\left(\phi_{+} A^{\mu} \phi_{-}+\phi_{-} A^{\mu} \phi_{+}\right) \\
& \mathcal{L}_{\pi \gamma \gamma}=\frac{F^{\pi}}{4} \epsilon^{\mu \nu \alpha \beta} \phi^{0} F_{\mu \nu} F_{\alpha \beta} \\
& \mathcal{L}_{\pi \pi \pi \gamma}=\frac{F^{3 \pi}}{6} \epsilon^{\mu \nu \alpha \beta} \epsilon^{a b c} A_{\mu} \partial_{\nu} \phi^{a} \partial_{\alpha} \phi^{b} \partial_{\beta} \phi^{c}
\end{aligned}
$$

In these expressions, $\Psi, \vec{\phi}, \Psi_{\Delta}, \Psi_{N^{*}}, \Psi_{N^{*}}, \vec{\phi}_{\mu}^{(\rho)}$ and $A_{\mu}$ stand for the nucleon, pion, $\Delta(1232), N^{*}(1440), N^{*}(1520), \rho(770)$ and photon fields, respectively; $m$ and $\mu$ are the nucleon and pion masses.

For the $\Delta \Delta \gamma$ coupling we write directly the vertex contribution to the Feynman rules [35] in analogy to the $N N \gamma$ case:

$$
-i \delta H_{\Delta \Delta \gamma}=i\left\{\frac{\left(\vec{p}+\vec{p}^{\prime}\right)}{2 m_{\Delta}} e_{\Delta}+i \frac{e \mu_{\Delta}}{3 m}\left(\vec{S}_{\Delta} \times \vec{k}\right)\right\} \cdot \vec{\varepsilon}
$$


where $\vec{S}_{\Delta}$ is the ordinary spin matrices for a spin $3 / 2$ object, $\vec{p}$ and $\vec{p}^{\prime}$ stand for the initial and final delta momentum respectively, and $\vec{k}$ for the photon momentum; $\mu_{\Delta}$ and $e_{\Delta}$ are the magnetic moment and charge of the delta; $m_{\Delta}$ is the delta mass.

The coupling constants are listed below.

\section{Coupling constants:}

$$
\begin{aligned}
& f=1 \\
& f^{*}=2.13 \\
& \lambda_{1}=0.0075 \\
& f_{\Delta}=0.802 \\
& f_{\Delta N \gamma}=0.116 \\
& \tilde{f}=0.477 \\
& C=-2.29 \mu^{-1} \\
& g_{\Delta N^{*} \pi}=2.07 \\
& e=0.3027 \\
& \tilde{f}_{N^{\prime *} \Delta \pi}=0.911 \\
& \tilde{g}_{N^{\prime *} \Delta \pi}=-0.552 \\
& \chi_{N}= \begin{cases}1.79 & \text { for proton } \\
-1.91 & \text { for neutron }\end{cases} \\
& \tilde{f}_{\gamma}^{N}= \begin{cases}0.0173 & \text { for proton } \\
-0.0112 & \text { for neutron }\end{cases} \\
& \tilde{g}_{\gamma}^{N}= \begin{cases}0.108 & \text { for proton } \\
-0.129 & \text { for neutron }\end{cases} \\
& \tilde{g}_{\sigma}^{N}= \begin{cases}-0.049 & \text { for proton } \\
0.0073 & \text { for neutron }\end{cases} \\
& G_{N N \rho}^{V}=2.9 \\
& G_{N N \rho}^{T}=18.15 \\
& f_{\rho}=6.14 \\
& C_{\rho}=2 \\
& \tilde{g}_{\rho}=0.591 \\
& g_{\rho \pi \gamma}=0.0378 \\
& F^{3 \pi}=0.0259 \mu^{-3} \\
& F^{\pi}=0.0035 \mu^{-1} \\
& \frac{\mu_{\Delta}}{\mu_{p}}=\frac{e_{\Delta}}{e} \\
& \mu_{\Delta^{++}}=1.62 \mu_{p}
\end{aligned}
$$

The magnetic moment of the $\Delta(1232), \mu_{\Delta}$, can be calculated in the quark model [36], with the result $\mu_{\Delta} / \mu_{p}=e_{\Delta} / e$. We shall use this result, except for the $\Delta^{++}$ where we shall use the experimental value $\mu_{\Delta^{++}}=(1.62 \pm 0.18) \mu_{p}$ based on the $\pi p$ bremsstrahlung $\left(\pi^{+} p \rightarrow \pi^{+} p \gamma\right)$ [37. 


\section{Appendix B}

TOTAL CROSS SECTION.

The cross section for the $\gamma N \rightarrow \pi \pi N$ reaction is given by

$$
\begin{gathered}
\sigma=\frac{m}{\lambda^{1 / 2}\left(s, 0, m^{2}\right)} \frac{1}{(2 \pi)^{5}} \int \frac{d^{3} p_{4}}{2 \omega_{4}} \int \frac{d^{3} p_{5}}{2 \omega_{5}} \int d^{3} p_{2} \frac{m}{E_{2}} \\
\delta^{4}\left(k+p_{1}-p_{2}-p_{4}-p_{5}\right) \overline{\sum_{s_{i}}} \sum_{s_{f}}|T|^{2} \\
=\frac{m^{2}}{\lambda^{1 / 2}\left(s, 0, m^{2}\right)} \frac{1}{4(2 \pi)^{4}} \int d \omega_{5} d \omega_{4} d \cos \theta_{5} d \phi_{45} \\
\theta\left(1-\cos ^{2} \theta_{45}\right) \overline{\sum_{s_{i}}} \sum_{s_{f}}|T|^{2}
\end{gathered}
$$

Where $k=(\omega, \vec{k}), p_{1}=\left(E_{1}, \vec{p}_{1}\right), p_{2}=\left(E_{2}, \vec{p}_{2}\right), p_{4}=\left(\omega_{4}, \vec{p}_{4}\right), p_{5}=\left(\omega_{5}, \vec{p}_{5}\right)$ are the momenta of the photon, incident proton, outgoing proton and the outgoing pions respectively. In (B.2) $\phi_{45}, \theta_{45}$ are the azimuthal and polar angles of $\vec{p}_{4}$ with respect to $\vec{p}_{5}$ and $\theta_{5}$ is the angle of $\overrightarrow{p_{5}}$ with the $z$ direction defined by the incident photon momentum $\vec{k}$. T is the invariant matrix element for the reaction. We reproduce this formula here since in Eq. (2) of ref. [11] there was a missprint and $d \theta_{5}$ should have been $d \cos \theta_{5}$, as in Eq. (B.2) above. 


\section{References}

[1] Aachen-Berlin-Bonn-Hamburg-Heidelberg-München collaboration, Phys. Rev. 175 (1968) 1669.

[2] G. Gialanella et al., Nuovo Cimento LXIII A (1969) 892.

[3] F. Carbonara et al., Nuovo Cimento 36A (1976) 219.

[4] A. Piazza et al., Nuovo Cimento III (1970) 403.

[5] Cambridge Bubble Chamber Group, Phys. Rev. 155 (1967) 1477; Phys. Rev. 163 (1967) 1510.

[6] H.R. Crouch et al., Phys. Rev. Lett. 13 (1964) 636; Phys. Rev. Lett. 13 (1964) 640.

[7] H.G. Hilpert et al., Phys. Lett. 23 (1966) 707.

[8] A. Braghieri, L. Murphy et al., to be published.

[9] H. Ströher, private communication.

[10] L. Lüke and P. Söding, Springer Tracts in Modern Physics 59 (1971) 39.

[11] J.A. Gómez Tejedor and E. Oset, Nucl. Phys. A571 (1994) 667.

[12] J. A. Gómez Tejedor, Tesina de Licenciatura, Universidad de Valencia, 1993.

[13] M. Benmerrouche and E. Tomusiak, Phys. Rev. Lett., 73 (1994) 400.

[14] V. Bernard, N. Kaiser, Ulf-G. Meißner and A. Schmidt, Nucl. Phys. A580 (1994) 475.

[15] V. Bernard, N. Kaiser and Ulf-G. Meißner, Phys. Rev. Lett. 74 (1995) 1036.

[16] M. Benmerrouche and E. Tomusiak, Phys. Rev. Lett. 74 (1995) 1037.

[17] J.A. Gómez Tejedor, M.J. Vicente-Vacas and E. Oset, Nucl. Phys. A588 (1995) 819.

[18] E. Oset and M.J. Vicente-Vacas, Nucl. Phys. A454 (1986) 637.

[19] N. Grion et al., Nucl. Phys. A492 (1989) 509.

[20] Particle Data Group, Phys. Rev. D50 (1994) 1173. 
[21] T.E.O. Ericson and W. Weise, Pions and Nuclei, Clarendon Press, (Oxford, 1988).

[22] R.C. Carrasco and E. Oset, Nucl. Phys. A536 (1992) 445.

[23] T.D. Cohen, Phys. Lett. B233 (1989) 467.

[24] Particle Data Group, Phys. Rev. D45 (1992).

[25] D.M. Manley and E.M. Saleski, Phys. D45 (1992) 4002.

[26] B.H. Bransden and R.G. Moorhouse, The Pion-Nucleon System, Princeton University Press, (1973).

[27] D.M. Manley, R.A. Arndt, Y. Goradia and V.L. Teplitz, Phys. Rev. D30 (1984) 904.

[28] E. Oset, talk given at XVth European Conference on few-Body Problems in Physics, in peñíscola (Spain), June 1995.

[29] E. Oset, talk given at International Conference on Mesons and light nuclei, in Straz (Czech Republic), July 1995.

[30] J.A. Gómez Tejedor, F. Cano and E. Oset preprint nucl-th/9510007.

[31] Benmerrouche et al. Phys. Rev. C39 (1989) 2339.

[32] V. Bernard, N. Kaiser and Ulf-G. Meißner, preprint hep$\mathrm{ph} / 9507418$.

[33] R.D. Amado, Modern three-hadron physics, ed. A.W. Thomas (Springer, Berlin, 1977).

[34] M.G. Olsson, Nucl. Phys. B78 (1974) 55.

[35] L. Heller, S. Kumano, J. C. Martínez and E. J. Moniz, Phys. Rev. C35 (1987) 718.

[36] F. E. Close, An introduction to quarks and partons, Ed. Academic Press, London, 1979.

[37] A. Bosshard et al., Phys. Rev. D44 (1991) 1962. 


\section{Figure captions:}

Fig. 1: Classification of the Feynman diagrams into one point, two point and three point diagrams. Continuous straight lines: baryons. Dashed lines: pions. Wavy lines: photons and $\rho$-mesons (marked explicitly).

Fig. 2: Total cross section for the $\gamma p \rightarrow \pi^{+} \pi^{-} p$ reaction. (a) $\tilde{f}_{N^{\prime *} \Delta \pi}=0.911$, $\tilde{g}_{N^{\prime *} \Delta \pi}=-0.552$; (b) $\tilde{f}_{N^{\prime *} \Delta \pi}=-0.911, \tilde{g}_{N^{\prime *} \Delta \pi}=0.552$.

Fig. 3: Feynman diagrams for the $\gamma p \rightarrow \pi^{+} \pi^{0} n, \gamma p \rightarrow \pi^{0} \pi^{0} p, \gamma n \rightarrow \pi^{+} \pi^{-} n$, $\gamma n \rightarrow \pi^{-} \pi^{0} p$ and $\gamma n \rightarrow \pi^{0} \pi^{0} n$ reactions.

Fig. 4: Feynman diagrams for the $\gamma N \rightarrow \pi^{0} N$ amplitude.

Fig. 5: Total cross section for the $\gamma p \rightarrow \pi^{+} \pi^{-} p$ reaction. Continuous line: total cross section. Short-dashed line: contribution of $\Delta(1232)$-intermediate states. Long-dashed line: contribution of $N^{*}(1520)$-intermediate state. Short-dash-dotted line: contribution of $N$-intermediate states. Long-dash-dotted line: contribution of $\rho$-intermediate states. Short-dash-long-dashed line: rest of the diagrams. Experimental data from refs. [1], 2, 3, 4, 8].

Fig. 6: Total cross section for the $\gamma p \rightarrow \pi^{+} \pi^{0} n$ reaction. Continuous line: total cross section. Short-dashed line: contribution of $\Delta(1232)$-intermediate states (diagrams (i)-(o) of Fig. 3). Long-dashed line: contribution of $N^{*}(1520)$-intermediate state (diagram (p) of Fig. 3). Short-dash-dotted line: contribution of $N$ - and $\rho$ intermediate states (diagrams (a)-(h) of Fig. 3). Long-dash-dotted line: contribution of $N^{*}(1440)$-intermediate states (diagrams (q)-(t) of Fig. 3). Experimental data from ref. [8].

Fig. 7: Total cross section for the $\gamma p \rightarrow \pi^{0} \pi^{0} p$ reaction. Continuous line: total cross section. Short-dashed line: contribution of $\Delta(1232)$-intermediate states (diagrams (i)-(o) of Fig. 3). Long-dashed line: contribution of $N^{*}(1520)$-intermediate state (diagram (p) of Fig. 3). Short-dash-dotted line: contribution of $N$-intermediate states (diagrams (a)-(g) of Fig. 3). Long-dash-dotted line: contribution of $N^{*}(1440)$ intermediate states (diagrams (q)-(t) of Fig. 3). Experimental data from ref. [8].

Fig. 8: Total cross section for the $\gamma n \rightarrow \pi^{+} \pi^{-} n$ reaction. Continuous line: total cross section. Short-dashed line: contribution of $\Delta(1232)$-intermediate states (diagrams (i)-(o) of Fig. 3). Long-dashed line: contribution of $N^{*}(1520)$-intermediate state (diagram (p) of Fig. 3). Short-dash-dotted line: contribution of $N$ - and $\rho$ intermediate states (diagrams (a)-(h) of Fig. 3). Long-dash-dotted line: contribution of $N^{*}(1440)$-intermediate states (diagrams (q)-(t) of Fig. 3). Experimental

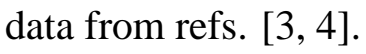


Fig. 9: Total cross section for the $\gamma n \rightarrow \pi^{-} \pi^{0} p$ reaction. Continuous line: total cross section. Short-dashed line: contribution of $\Delta(1232)$-intermediate states (diagrams (i)-(o) of Fig. 3). Long-dashed line: contribution of $N^{*}(1520)$-intermediate state (diagram (p) of Fig. 3). Short-dash-dotted line: contribution of $N$ - and $\rho$ intermediate states (diagrams (a)-(h) of Fig. 3). Long-dash-dotted line: contribution of $N^{*}(1440)$-intermediate states (diagrams (q)-(t) of Fig. 3). Experimental

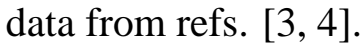

Fig. 10: Total cross section for the $\gamma n \rightarrow \pi^{0} \pi^{0} n$ reaction. Continuous line: total cross section. Short-dashed line: contribution of $\Delta(1232)$-intermediate states (diagrams (i)-(o) of Fig. 3). Long-dashed line: contribution of $N^{*}(1520)$-intermediate state (diagram (p) of Fig. 3). Short-dash-dotted line: contribution of $N$-intermediate states (diagrams (a)-(g) of Fig. 3). Long-dash-dotted line: contribution of $N^{*}(1440)$ intermediate states (diagrams $(\mathrm{q})$ and $(\mathrm{t})$ of Fig. 3).

Fig. 11: Total cross section for the $\gamma p \rightarrow \pi^{0} \pi^{0} p$ reaction. (a) $\tilde{f}_{N^{\prime *} \Delta \pi}=0.911$, $\tilde{g}_{N^{\prime *} \Delta \pi}=-0.552$; (b) $\tilde{f}_{N^{\prime *} \Delta \pi}=-0.911, \tilde{g}_{N^{\prime *} \Delta \pi}=0.552$.

Fig. 12: Total cross section for the $\gamma p \rightarrow \pi^{+} \pi^{-} p$ reaction at energies close to threshold. Continuous line: results of our model. Long-dashed line: results of $\mathrm{M}$. Benmerrouche and E. Tomusiak [13]. Short-dashed line: results of V. Bernard et al. [14].

Fig. 13: Total cross section for the $\gamma p \rightarrow \pi^{0} \pi^{0} p$ reaction at energies close to threshold. Continuous line: results of our model. Long-dashed line: results of $\mathrm{M}$. Benmerrouche and E. Tomusiak [13]. Short-dashed line: results of V. Bernard et al. [14]. 


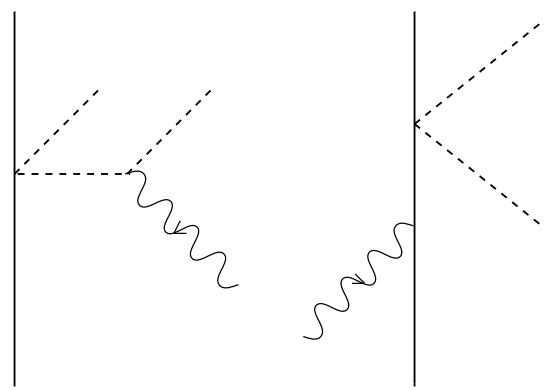

(a)

(b)

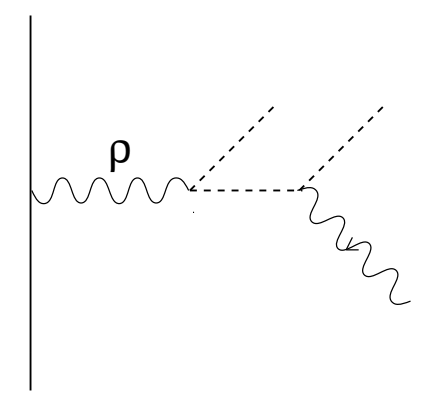

(f)

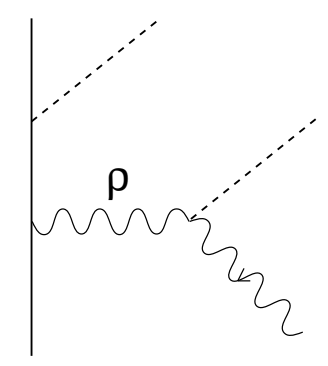

(i)

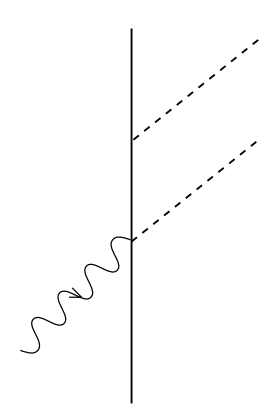

(c)

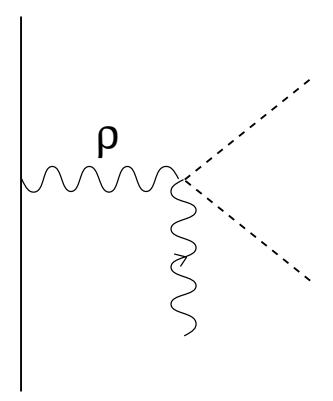

(g)

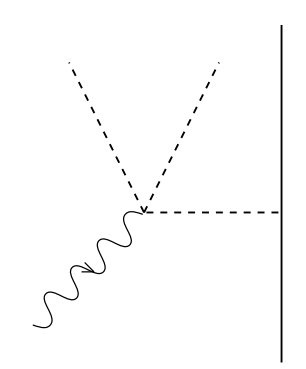

(j)

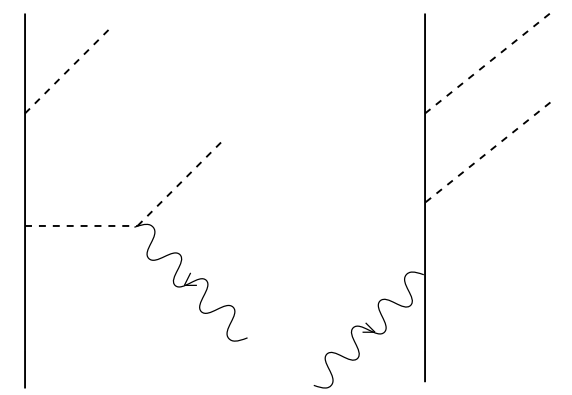

(e)

(d)

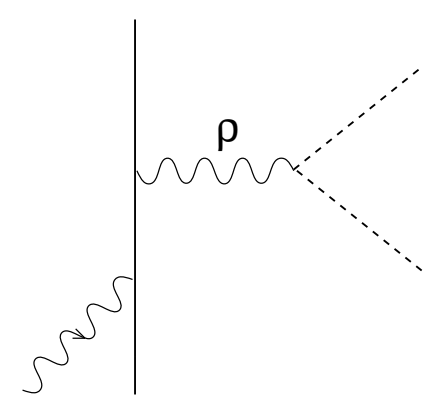

(h)

Fig. 1 


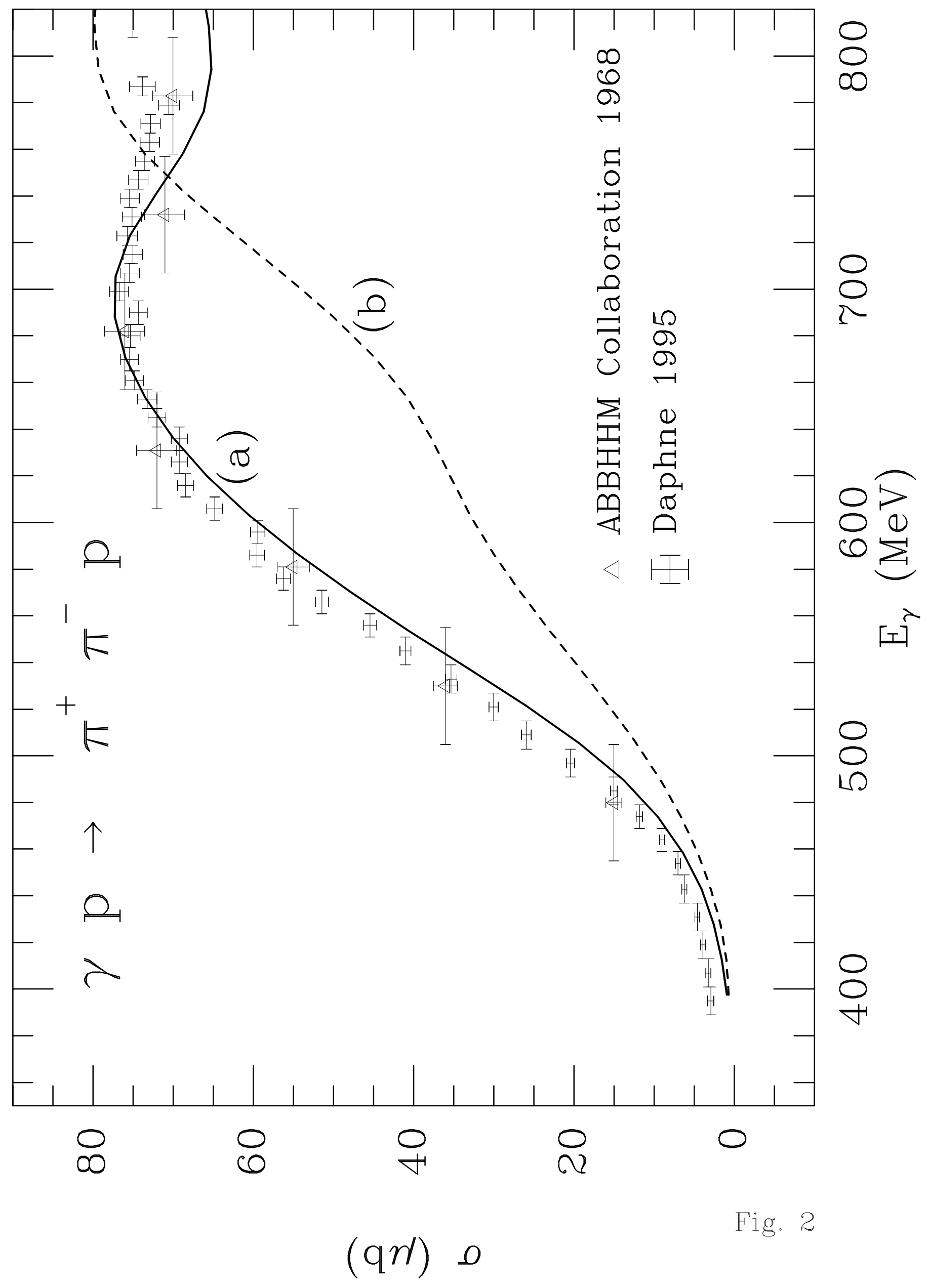




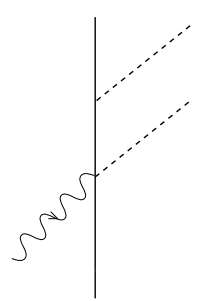

(a)

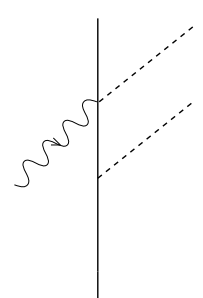

(b)

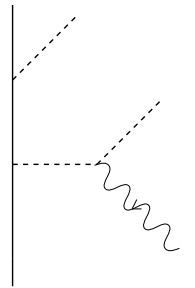

(c)

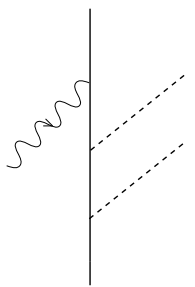

(g)

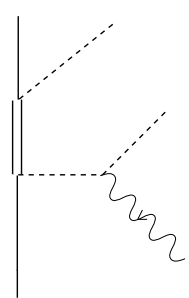

(k)

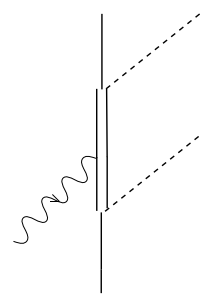

(o)

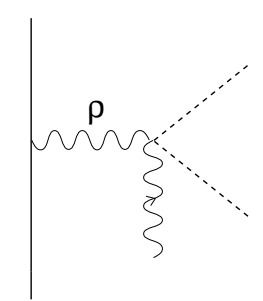

(h)

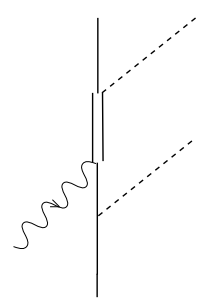

(I)

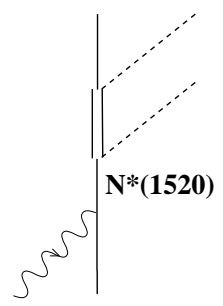

(p)

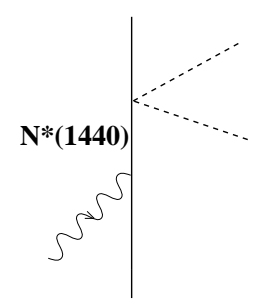

(q)

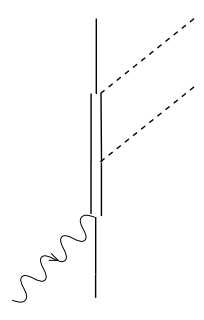

(n)

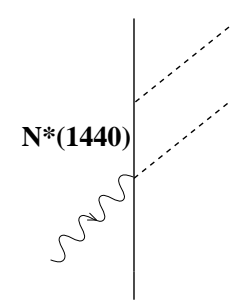

(r)

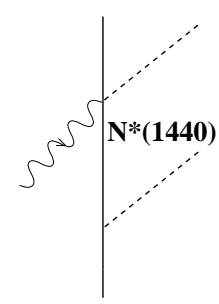

(s)

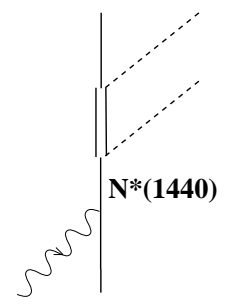

(t)

Fig. 3 


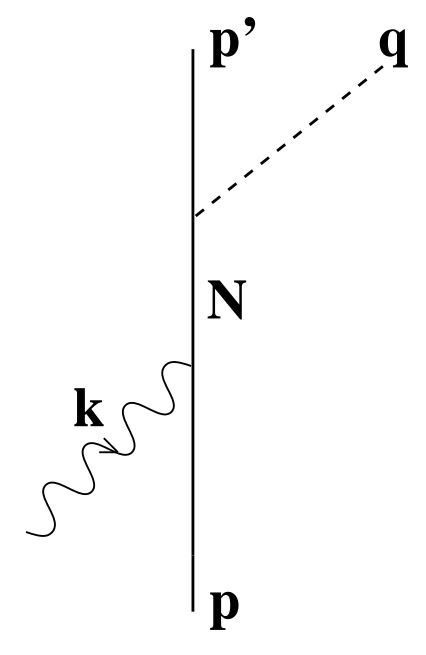

(a)

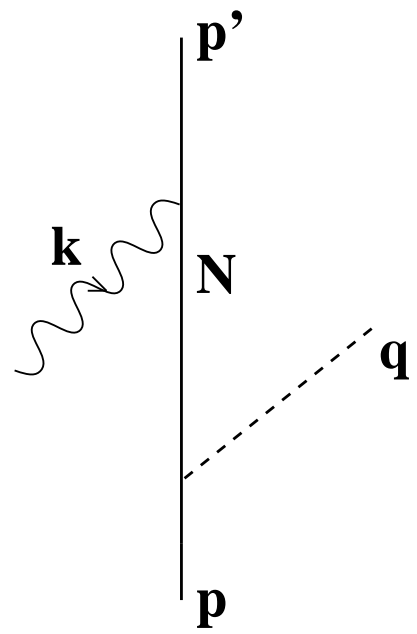

(b)

Fig. 4 


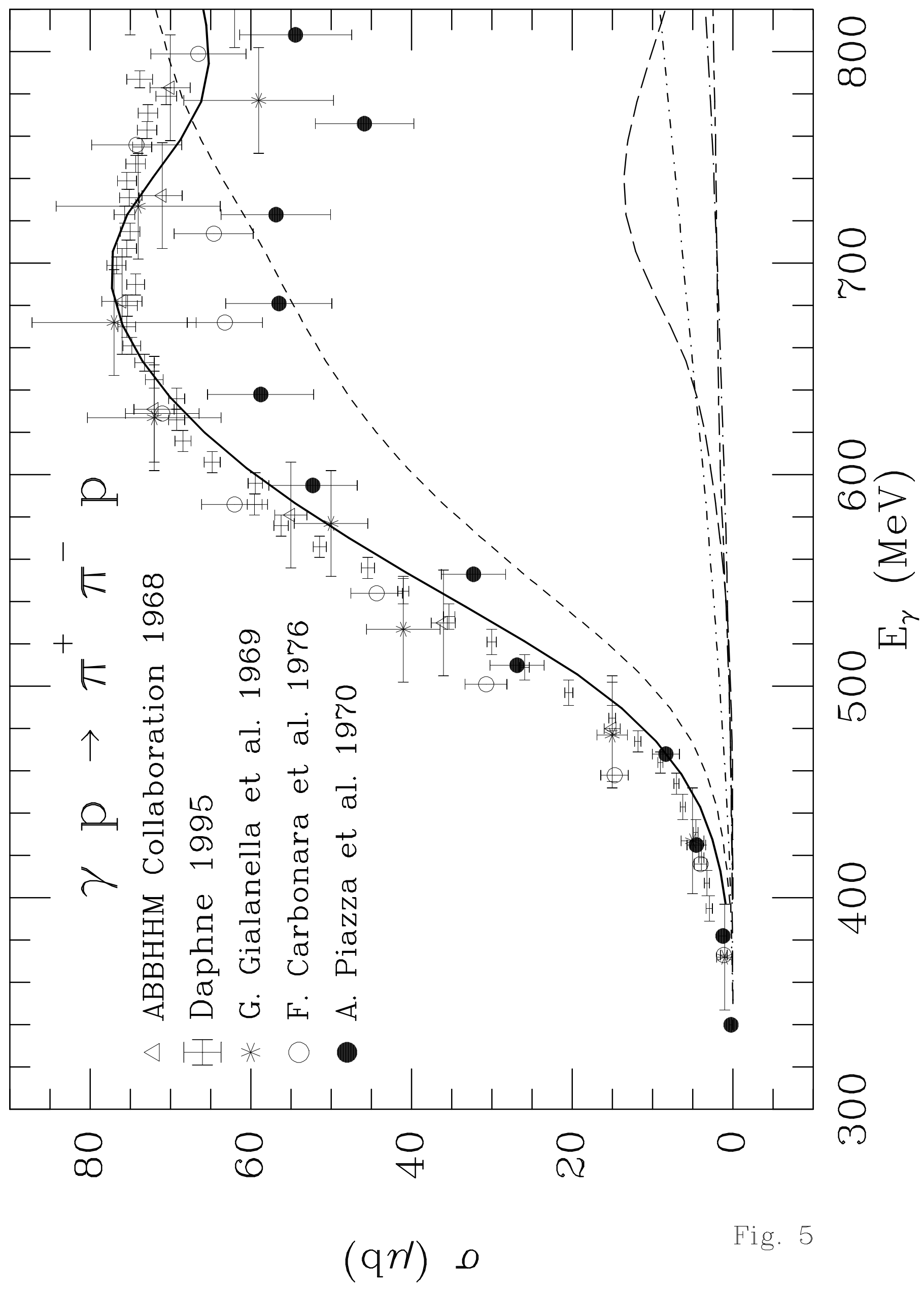




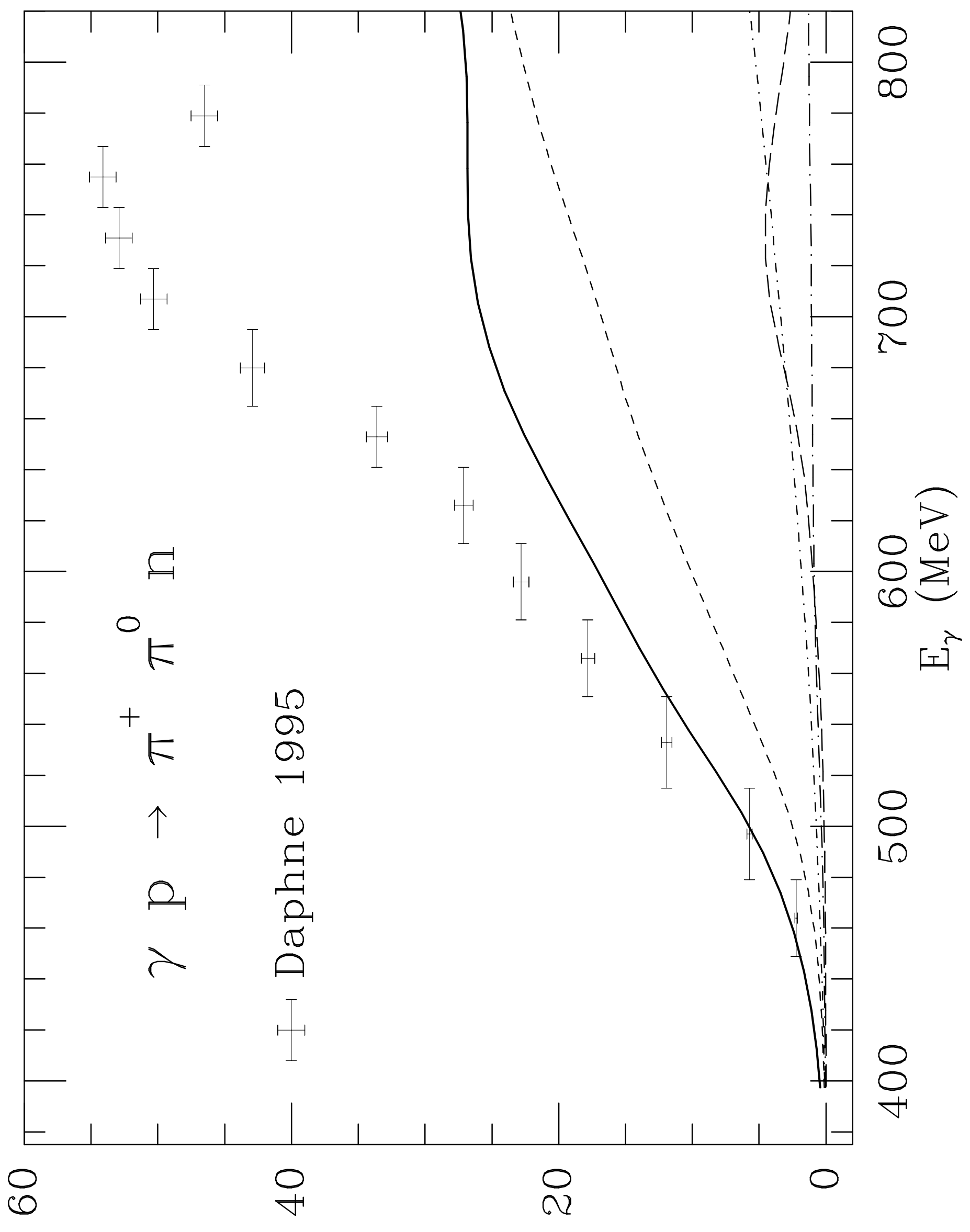

$(q r) \rho$

Fig. 6 


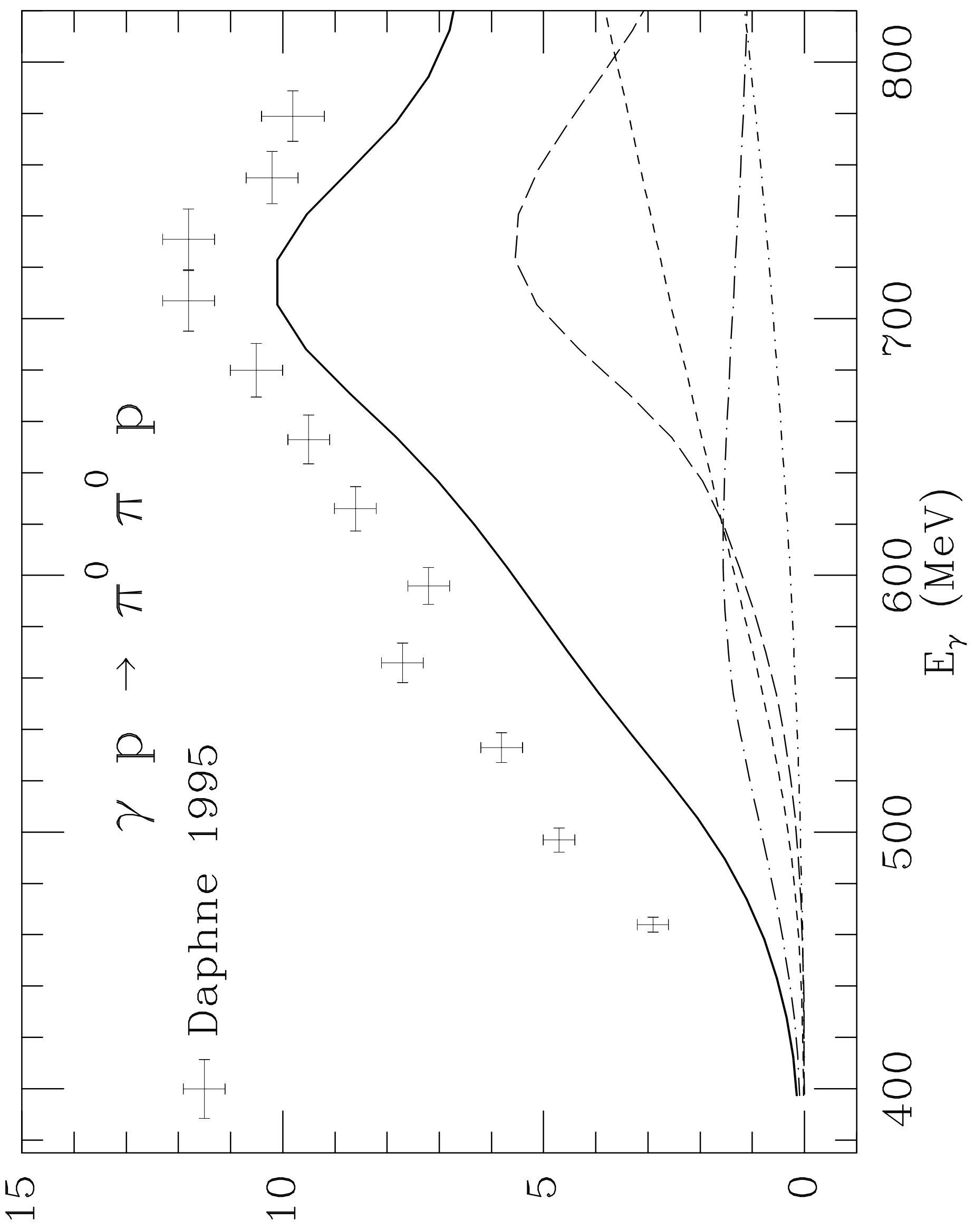

$(\mathrm{q} r) \rho$

Fig. 7 


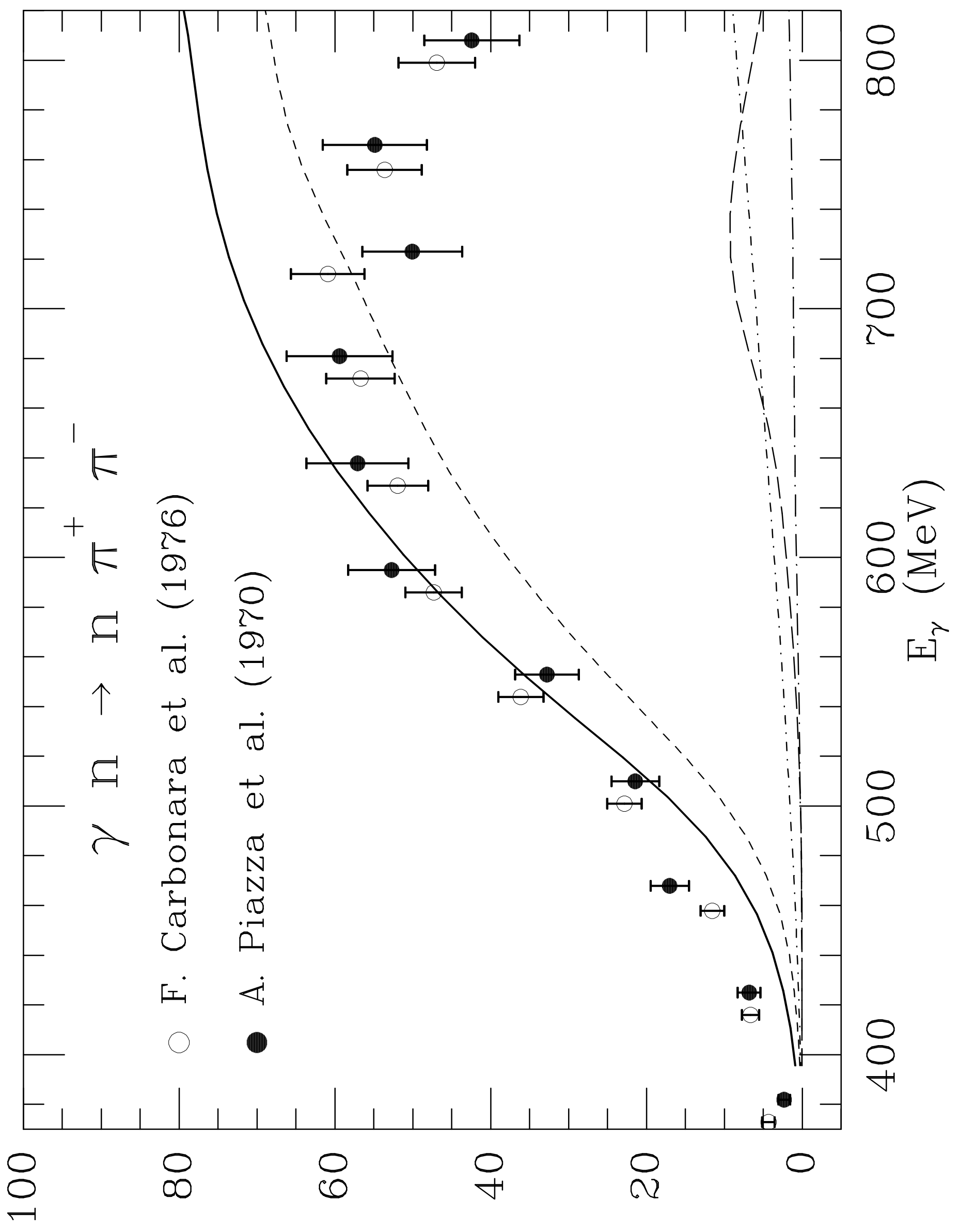

$(\mathrm{q} r) \rho$

Fig. 8 


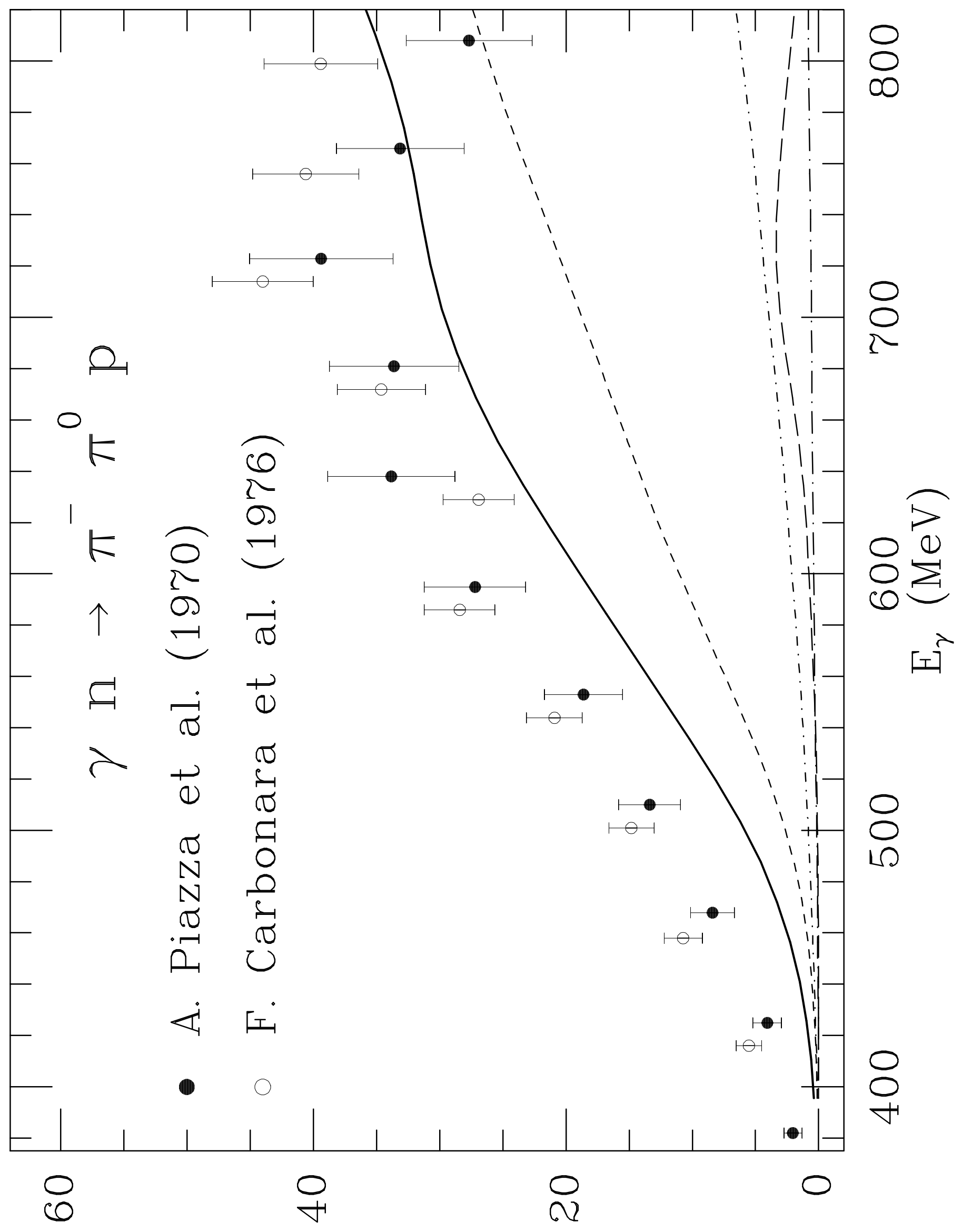

$(q r) \rho$

Fig. 9 


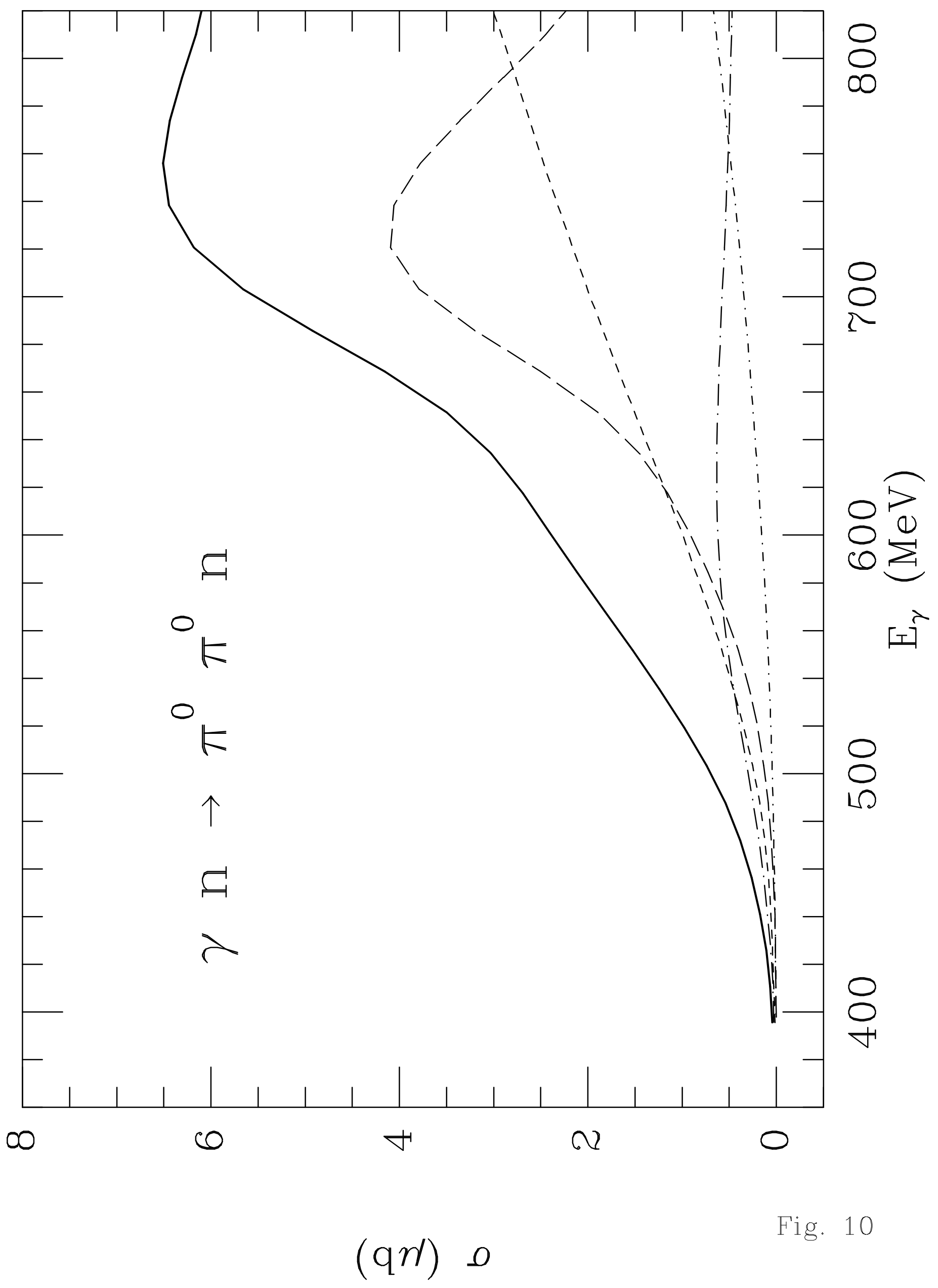




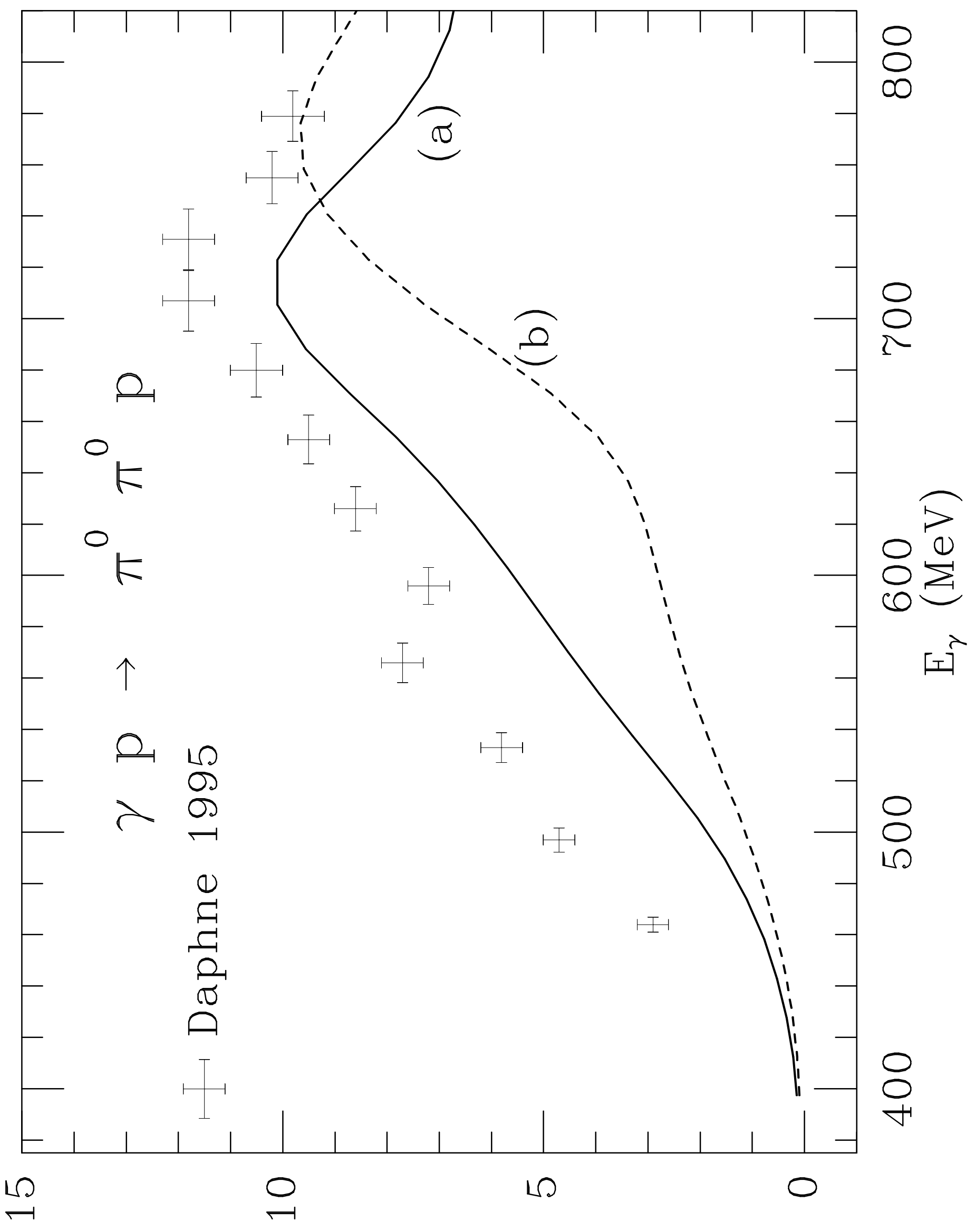

$(\mathrm{q} r) \rho$

Fig. 11 


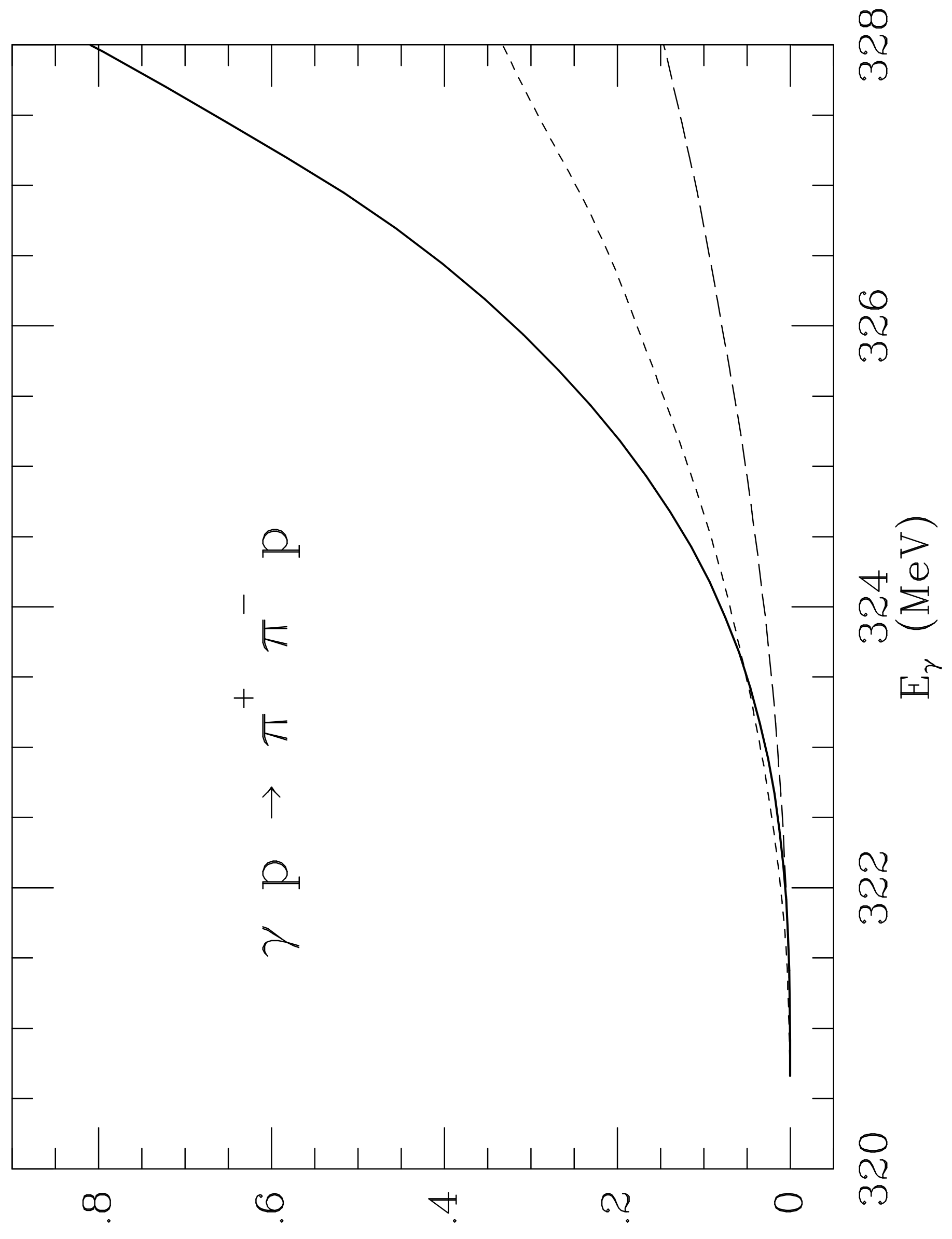

(qu) $\triangle$

Fig. 12 


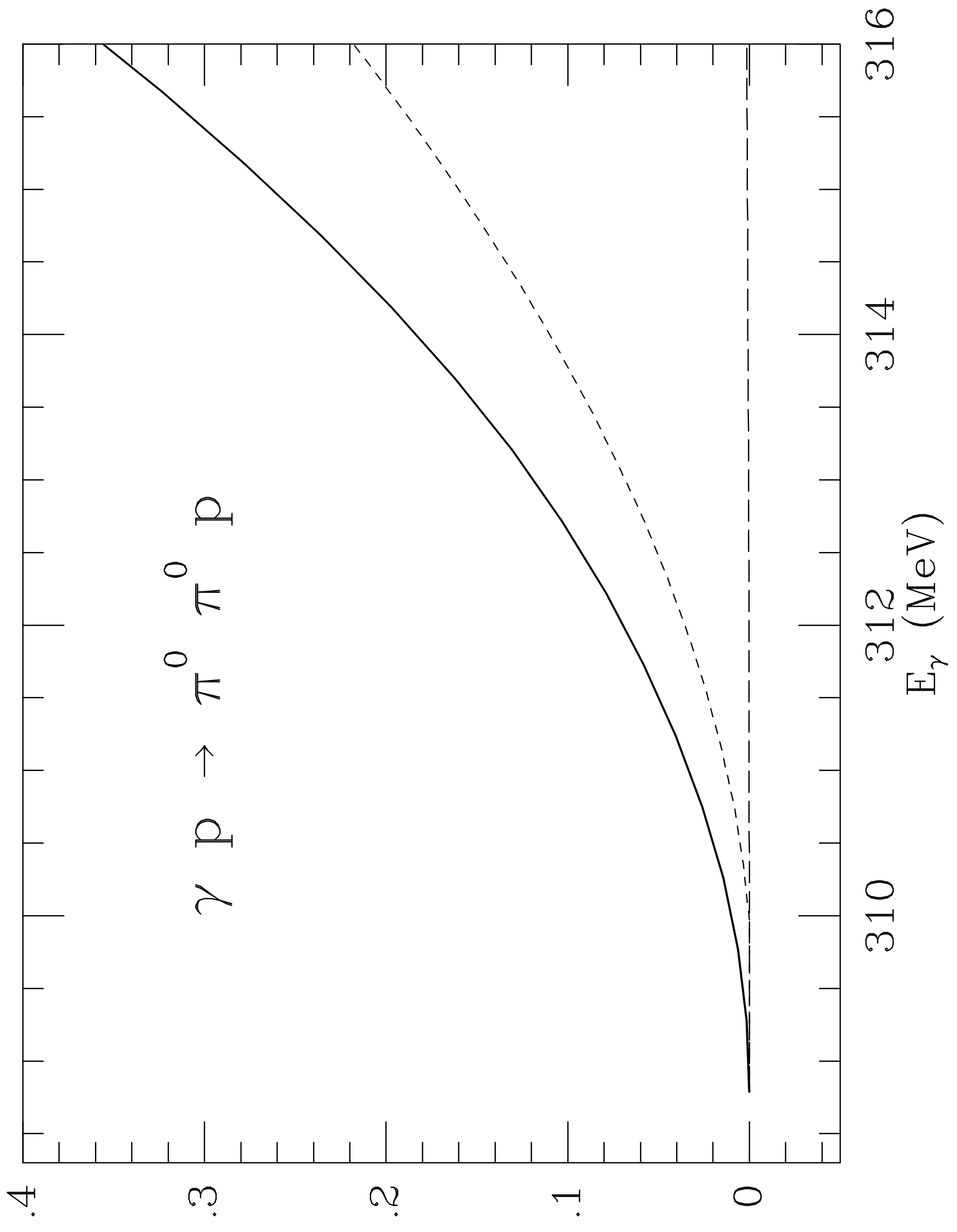

(qu) $\triangle$

Fig. 13 